\title{
ATLAST-8 Mission concept study for 8-meter Monolithic UV/Optical Space Telescope
}

\author{
H. Philip Stahl ${ }^{\mathrm{a}}$, Marc Postman ${ }^{\mathrm{b}}$, William R. Arnold Sr. ${ }^{\mathrm{c}}$, Randall C. Hopkins ${ }^{\mathrm{a}}$, \\ Linda Hornsby ${ }^{\mathrm{c}}$, Gary E. Mosier ${ }^{\mathrm{d}}$, and Bert A. Pasquale ${ }^{\mathrm{d}}$ \\ ${ }^{a}$ NASA Marshall Space Flight Center, Huntsville, AL \\ ${ }^{\mathrm{b}}$ Space Telescope Science Institute, Baltimore, MD \\ ${ }^{\mathrm{c}}$ Jacobs ESTS Group, Marshall Space Flight Center, Huntsville, AL \\ ${ }^{\mathrm{d}}$ NASA Goddard Space Flight Center, Greenbelt, MD
}

\begin{abstract}
ATLAST-8m is an 8-meter monolithic UV/optical/NIR space observatory which could be placed in orbit at Sun-Earth L2 by a heavily lift launch vehicle. Two development study cycles have resulted in a detailed concept including a dual foci optical design; several primary mirror launch support and secondary mirror support structural designs; spacecraft propulsion, power and pointing control design; and thermal design. ATLAST-8m is designed to yield never before achieved performance to obtain fundamental astronomical breakthroughs.
\end{abstract}

Keywords: Large Space Telescopes, UV/Optical Space Telescopes, Astronomy, Heavy Lift Launch Vehicle

\section{INTRODUCTION}

ATLAST-8m is an 8-meter monolithic UV/optical/NIR space observatory which could be placed in orbit at Sun-Earth L2 by a heavy lift launch vehicle with capabilities similar to the NASA Ares V cargo launch vehicle. $(1,2,3)$ ATLAST-8m is one of three mission concepts (ATLAST-8m, ATLAST-9m and ATLAST-16m) studied by the Astrophysics Mission Concept Study Advanced Technology Large-Aperture Space Telescope (ATLAST) effort lead by Marc Postman of the Space Telescope Science Institute (STScI). (4) ATLAST-8m started in 2006 as a 6 to 8 meter joint concept study between Marshall Space Flight Center (MSFC) and STScI. Preliminary results of this initial study were presented at the Tempe 2007 Science Associated with Lunar Exploration Architecture Meeting $(5,6)$ and several other conferences. $(7,8,9)$ Upon receiving the Astrophysics Concept Study in 2008, the ATLAST-8 team was expanded to include Goddard Space Flight Center (GSFC), Ball Aerospace Technology Corporation and Northrop Grumman.

The result of the Astrophysics Study is a detailed concept (4) with a dual foci optical design (10); primary mirror launch support and secondary mirror support structural design (11); spacecraft propulsion, power and pointing control design (12); and thermal design (13). ATLAST-8m is an 8-meter monolithic telescope with $50 \mathrm{~m}^{2}$ collecting aperture. Specific on-axis and off-axis configurations were explored. It assumes the same optical coatings as used on the Hubble Space Telescope for broadband UV/OIR (110 $\mathrm{nm}$ to $2400 \mathrm{~nm}$ ) performance. With a $10 \mathrm{~nm}$ rms primary mirror and an extremely stiff structure, ATLAST- $8 \mathrm{~m}$ is able to achieve a system level diffraction limited of $500 \mathrm{~nm}$. And, ATLAST- $8 \mathrm{~m}$ has sufficient pointing stability to take full advantage of its 16 milli-arc-sec (mas) angular resolution. The $10 \mathrm{~nm}$ rms primary mirror surface figure error is achieved because ATLAST-8m assumes a massive solid meniscus primary mirror (which can be polished with no quilting). And, because of the primary mirror thermal mass, ATLAST-8m has an extremely stable 500 hour thermal time constant. Innovative spacecraft design features enable 4500 to 9000 hours of pointing stability for long exposure observations. The combination of these engineering parameters results in a space observatory with never before achieved performance which will enable ATLAST- $8 \mathrm{~m}$ to study some of the most compelling science questions of our generation and yield fundamental astronomical breakthroughs. $(14,15)$ Additionally, a one year study was funded at the University of Alabama Huntsville to explore multiple robotic servicing options for ATLAST-8m.

Finally, it is true that ATLAST-8m is wholly dependent upon the existence of a heavy lift launch vehicle with capacities similar to the NASA Ares V vehicle, i.e. a 10 meter diameter by 23 meter tall fairing and $56 \mathrm{mT}$ mass to Sun-Earth L2 Transfer Orbit. But, given the length of time needed to design, build and launch space telescopes (26 years for Hubble, 24 years for Chandra, and at least 24 years for JWST), it is recommended that ATLAST-8m be developed in parallel with NASA's new heavy lift launch vehicle. Because of its low risk high technical level maturity, ATLAST-8m could be ready to launch by mid to late 2020s. After JWST, ATLAST-8m is ready to be the next 'Great Observatory' of the $21^{\text {st }}$ Century. 


\section{SCIENCE MOTIVATION}

The science case for ATLAST is compelling $(14,15)$. ATLAST- $8 \mathrm{~m}$ has the performance necessary to answer some of the most compelling astronomical questions, including: Is there life elsewhere in the Galaxy?; What is the underlying physics driving star formation?; and What are the complex interactions between dark matter, galaxies and the intergalactic medium? By virtue of its $\sim 15$ milli-arcsec (mas) angular resolution at $\sim 500 \mathrm{~nm}$ coupled with its ultra high sensitivity, superb stability and low sky background, ATLAST-8m will achieve major breakthroughs in astrophysics by enabling fundamentally new observations - both on its own and in combination with other telescopes with different capabilities. ATLAST-8m has the performance required to detect the potentially rare occurrence of bio-signatures in the spectra of terrestrial exo-planets, to reveal the underlying physics that drives star formation, and to trace the complex interactions between dark matter, galaxies, and the intergalactic medium. ATLAST- $8 \mathrm{~m}$ will be able to observe the habitable zone of 145 long-lived stars (spectral type F,G,K) up to $\sim 25$ parsecs (Figure 1). ATLAST-8m can determine planetary rotation rates, the presence of continents, oceans, and clouds as well as determine planetary habitability and the possible presence of life by obtaining spectra of ozone, molecular oxygen, atmospheric column density and Rayleigh scattering, and water vapor. The spectral features required to assess the above processes are all in the $300-2400 \mathrm{~nm}$ range and, hence, the exo-planet characterization instrument on ATLAST is sensitive at these wavelengths. ATLAST-8m will be able to study star formation histories in the local universe by measuring accurate colors and luminosities of stars in over 100 galaxies, of which over ten are giant spirals and one is a giant elliptical (Figure 2). And, it can study dark matter by determining subtle transverse motions of stars to measure density profiles in dwarf spherioid galaxies. ATLAST-8m will be able to study the relationship between galaxy formation and super massive black holes by obtaining spatially resolved images and spectra of the disks of accreting gas around 1740 super massive black holes out to a redshift of 0.36 . And, it will enable the study of galaxy and large structure formation by directly measuring key properties (both for dark and visible matter) of structures and mapping their evolution in time. Because of the large leap in observing capabilities that ATLAST-8m provides, one cannot fully anticipate the diversity or direction of the investigations that will dominate its use - just as the creators of HST did not foresee its pioneering roles in characterizing the atmospheres of Jupiter-mass exoplanets or measuring the acceleration of cosmic expansion using distant supernovae.

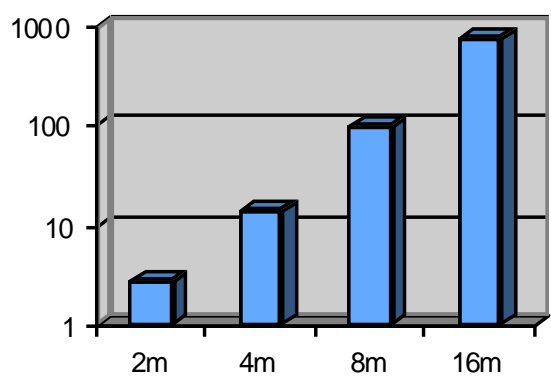

Figure 1: Average number of $\mathrm{F}, \mathrm{G}, \mathrm{K}$ stars where $\mathrm{SNR}=10 \mathrm{R}=70$ spectrum of an Earth-twin could be obtained in $<500 \mathrm{ksec}$ as a function of telescope diameter. Growth in the sample size scales as $\mathrm{D}^{3}$.
Figure 2: Map of local universe (24 Mpc across) shown with distances out to which HST (yellow), JWST (orange), and ATLAST (8-m, 16-m), can detect solar analogs in $\mathrm{V}$ and $\mathrm{I}$ pass-bands at $\mathrm{SNR}=5$ in 100 hours. Giant spirals, like M31, are indicated by blue galaxy symbols, giant ellipticals as orange blobs, and dwarf galaxies as green dots.

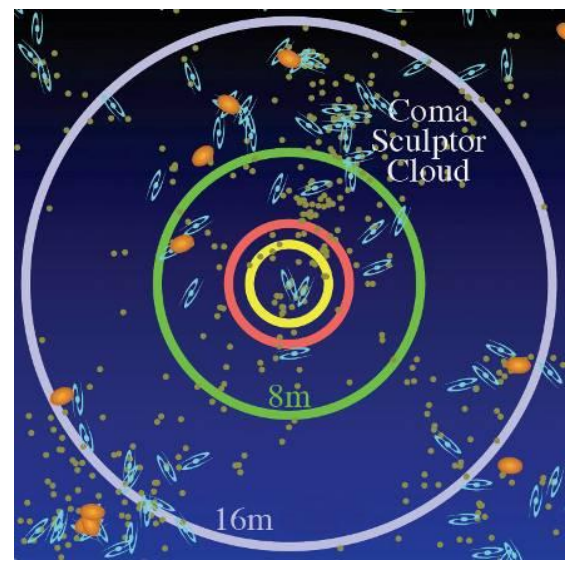

\section{MISSION CONCEPT}

ATLAST-8m is intended to be launched from KSC on a heavy lift launch vehicle with capabilities similar to the Ares V vehicle (configuration 51.01.48), which could place $65 \mathrm{mt}$ of payload onto a transfer orbit with a C3 energy of $-0.7 \mathrm{~km} / \mathrm{s}^{2}$. This energy is sufficient to send ATLAST-8m to a halo orbit about the second Sun-Earth Lagrange point (SE-L2) using a direct insertion trajectory $[2,3]$. While the NASA Constellation Program is in a state of flux, there appears to be a National commitment to design and build a heavy lift launch vehicle. Several current heavy lift design studies at MSFC are including the ATLAST-8m observatory in the trade space of potential payloads that define the desired capabilities of a future heavy lift launch vehicle. Preliminary results indicate that ATLAST-8m is not a driving element for any potential heavy lift vehicle. Beyond mass to orbit, the key capacity requirement which enables ATLAST-8m is launch faring volume. The ATLAST-8m concept requires a dynamic envelope of at least 8.8 meters diameter by 17 meters tall (Figure 3). The Ares V 10 meter diameter by 23 meter tall fairing provided this envelope. The $8.8 \mathrm{~m}$ diameter dynamic envelop allows an $8 \mathrm{~m}$ diameter primary mirror to be launched face up (which is best for launch survival purposes). The $17 \mathrm{~m}$ tall dynamic envelop allows for the secondary mirror to be launched fully deployed. This reduces risk and allows for a structurally stiff and stable optical bench and secondary mirror support structure. 
Once on-orbit, the 60 degree scarfed forward sunshield slides forward, the protective doors open, the solar panels deploy, and the optical component launch locks release (Figure 4). At this point the observatory is ready for commissioning and alignment of the optical system. The wavefront sensing and control system iteratively uses the star trackers and fine guidance sensors to point stars onto phase-diversity sensors distributed about the observatory field of view. Alignment is achieved by moving the secondary mirror via its hexapod and if necessary the tertiary mirror.

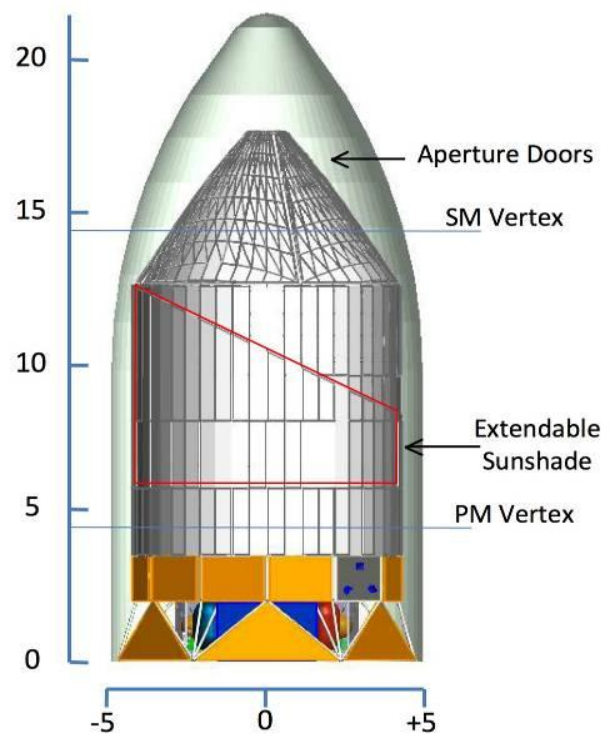

Figure 3: ATLAST-8m in its launch configuration in an Ares-V ogival fairing. The scarfed end of the sunshade is deployed once on-orbit. Length scales are in meters.

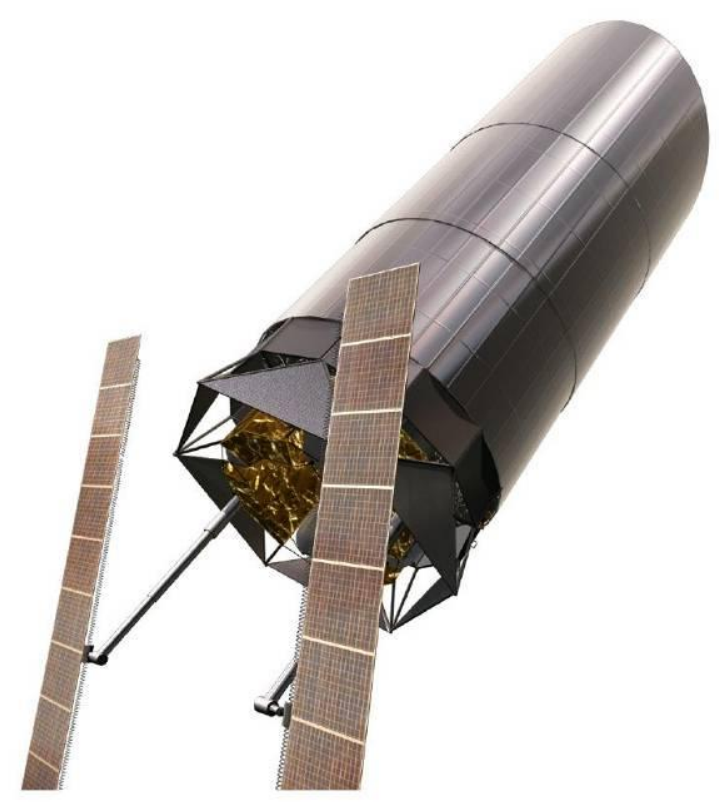

Figure 4: ATLAST-8m exterior view showing twin gimbaled solar arrays.

The size and geometry of the halo orbit can be traded during Phase A, but is assumed to be the JWST orbit. This orbit has the advantage of not requiring an insertion maneuver at SE-L2. Once at SE-L2 the spacecraft only has to begin station keeping duties to remain on the halo orbit. Available volume in the ATLAST-8 structure and mass margin makes it possible to add extra propellant tanks and carry a sufficient propellant load for a 20 year (or longer) mission. Analysis indicates that only $5 \mathrm{~m} / \mathrm{s}$ delta-v is needed per year for station keeping and momentum unloading. Given the 6-month period of the halo orbit and the 60-degree keep-out angle between the telescope's line of sight and the sun, the telescope can see the entire sky in approximately six months.

\section{OBSERVATORY DESIGN}

The fundamental philosophy of ATLAST- $8 \mathrm{~m}$ is to trade mass for simplicity. Instead of pursuing a complex design imposed by a launch vehicle mass and volume constraint, the design team used the unparalleled capabilities of NASA's potential Ares V launch vehicle to design a simple low risk observatory using high TRL (but massive) technology. Using mass reduces technical, programmatic and performance risk, and cost. A study of space telescope missions has discovered that telescopes without a launch mass constraint tended to be 10X more massive and $60 \%$ lower cost than those with a design to mass constraint [16]. Mass has performance advantages as well. A more massive telescope is stiffer. Therefore it is more mechanically and thermally stable. Also, it is easier to achieve exceptionally smooth optical surfaces on a solid mirror substrate. Finally, the volume of an Ares V class vehicle allows the launch of a fully deployed telescope.

The ATLAST Astrophysics Mission Concept Study developed a detailed point design for an 8-m monolithic observatory. Specific studies included: optical design; structural design/analysis including primary mirror support structure, sun shade and secondary mirror support structure; thermal analysis; spacecraft conceptual design including structure, propulsion, GN\&C, avionics, thermal and power systems; mass and power budgets; and system cost [4].

\subsection{Optical Telescope Assembly}

Optical Design: The optical telescope assembly (OTA) has a dual-field design with three foci (Figure 5). (10) All three foci are diffraction limited at $500 \mathrm{~nm}$. The main telescope is a two-mirror system, which forms a narrow-field-of-view (NFOV) 1 arc-min Cassegrain (CASS) image. The Cassegrain focus provides specific technical advantages for two classes of high-priority science. It provides a high quality NFOV focus for exo-planet characterization science, and it provides a 
high-throughput two-bounce path for UV spectroscopy science. The primary and secondary mirrors' optical coatings are identical to what was used by HST: aluminum with MgF2 overcoat to provide good spectral transmission from $110 \mathrm{~nm}$ to $2400 \mathrm{~nm}$. These coatings are important to the UV science instruments at Cassegrain focus. Two pick-off fold mirrors, on either side of CASS focus, direct off-axis portions of the Cassegrain image plane to two tertiary-mirror aft-optics assemblies, which form two wide-field-of-view (WFOV) $8 \times 22$ arc-min three-mirror anastigmatic (TMA) images. The TMA provides a 13 mas plate scale. The aft optics are coated with protected silver as used on Kepler for enhanced visible/near-ir spectral transmission. All three foci are directly accessible to a $4.0 \mathrm{~m}$ diameter by $4.5 \mathrm{~m}$ deep instrument bay centered on-axis behind the primary mirror.

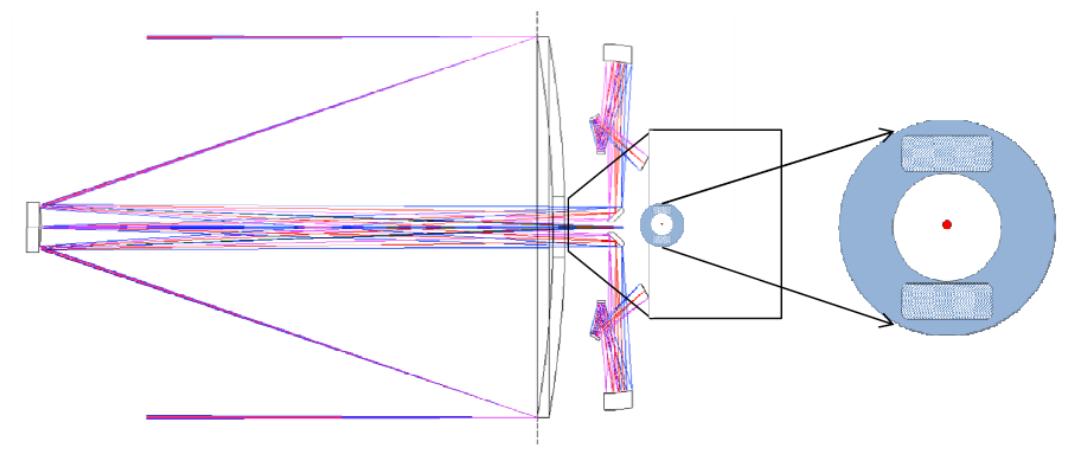

Figure 5: Optical Layout of 8-m OTA showing 2 TMA foci and Cass focus (at red dot)

Additionally, for exo-planet science, an off-axis dual-field optical design was developed. Conventional internal Lyot stop coronagraphs desire a 'clean' point spread function without diffraction from secondary mirror support structure. The resultant design has two potential problems. First, because of the size of the secondary mirror and the need to keep its shadow clear of the primary mirror, the maximum primary mirror aperture which can be implemented is approx 6x8 meters. Second, to keep the primary mirror surface aspheric departure slope and alignment tolerances reasonable, the secondary to primary mirror distance is 12 meters. Given current launch faring sizes, this design would require a deployed secondary.

Primary Mirror: The single most important element of ATLAST-8m is the primary mirror. While a HST-style or (proposed TPF-C style) lightweight mirror could be used with a JWST-class design-margin structure, the mass capacity of a potential heavy lift launch vehicle allows us to reduce risk and cost by using a solid meniscus glass mirror - the kind typically used for ground based telescopes (e.g., Gemini, VLT and Subaru). Such mirrors offer several significant advantages for a space-based telescope. They are extremely thermally stable, mechanically very stiff, and they cost effectively achieve a very smooth optical surface. These are all properties that are important for ultra-high contrast imaging. Both REOSC and Brashear have demonstrated the ability to fabricate $8 \mathrm{~m}$ class mirrors to better than $10 \mathrm{~nm} \mathrm{rms}$. This fact highlights another important advantage of the proposed mirror: the infrastructure exists and is proven for handling, manufacturing, and testing $8 \mathrm{~m}$ class mirrors. Currently there is one existing $8 \mathrm{~m}$ Zerodur mirror blank at Schott Glass in Germany and Corning has a proven furnace capability to make ULE glass mirror blanks.

The secondary mirror can be made of ULE or Zerodur. While the SM could be a lightweight mirror, the baseline is a solid meniscus. Again, the advantage of a solid meniscus is a lower cost and smother mirror than can be achieved with a lightweighted mirror. A lightweighted mirror will only be considered if analysis shows that reducing SM assembly mass is required to meet launch environment design safety margins. A solid meniscus mirror also enables the ability to certify its convex aspheric surface figure using a through the back test. The SM assembly employs a six-degree-of- freedom hexapod virtually identical to the one to be used on JWST.

Science Instruments: A notional concept has five science instruments and two facility instruments (Figure 6). The exoplanet instrument and UV spectrometer are at CASS focus. The WFOV imager, multi-object spectrograph and IFU spectrograph are divided between the two TMA focal planes. Additionally, each of the three foci has wavefront sensors and each TMA focus has two Fine Guidance Sensors (FGS). Each instrument module is a self-contained On-orbit Replaceable Unit (ORU) using HST-style mounting rails accessible from the back of the instrument bay to facilitate servicing missions. The instrument bay provides all required mechanical, electrical, data and thermal interface connections for the science modules using standard HST-style 'blind-mate' connectors. Heat-pipe connections scavenge instrument heat to help maintain the OTA at $280 \mathrm{deg} \mathrm{K}$ and route excess heat to external radiators. The spacecraft envelope surrounds the instrument bay, which is isolated from both the primary mirror support structure and the instrument bay. The Instrument Command and Data Handling unit (IC\&DH) provides centralized OTA electronics for control of telescope mechanisms and heaters, wavefront sensing (WFS) processors, and science instruments. 


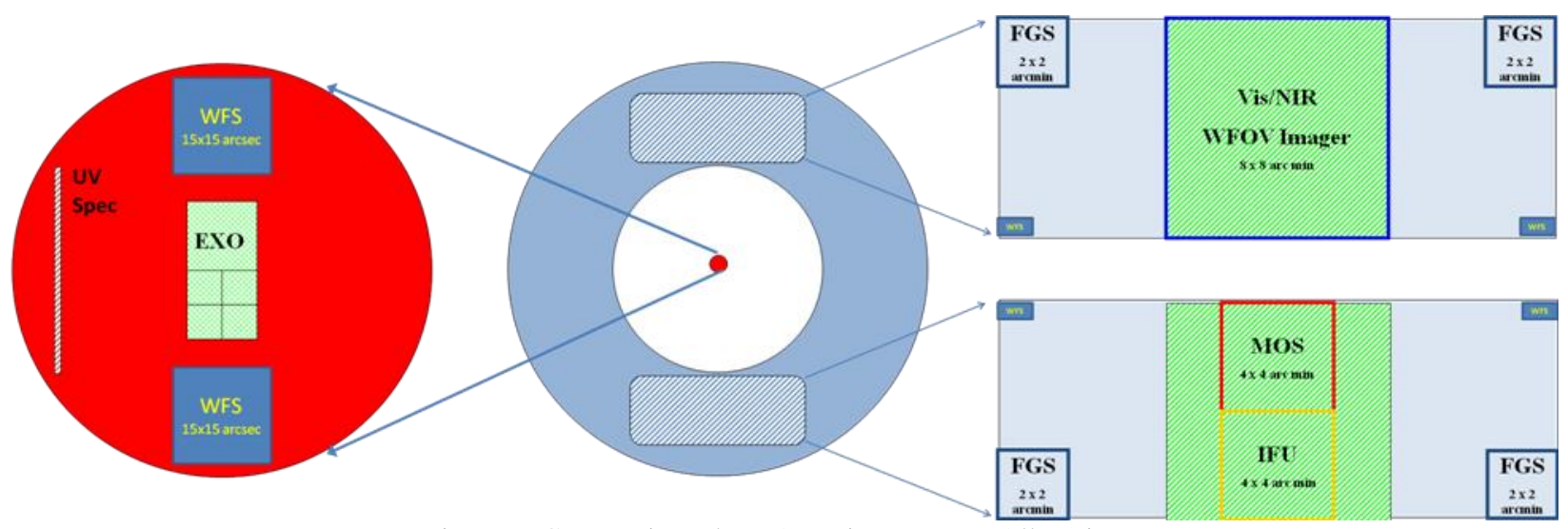

Figure 6: Cassegrain and TMA Foci Instrument Allocations.

The two TMA FOVs are separated on the sky by approximately 0.5 degrees. This separation helps the FGS pointing system control roll well enough to meet the 1.6 mas pointing stability requirement. Separating the two WFOV instruments allows added flexibility in packaging as well as future functionality after a servicing mission.

Structure: The ATLAST-8m structure contains several major elements (Figure 7): primary mirror support structure, metering truss, instrument bay, secondary mirror spiders, aft optics structure and payload adapter fixture. (11) All structural elements are fabricated from a cyanate ester graphite epoxy composite material with flight certified thermal and mechanical properties. The structure is divided between the forward and back structure.
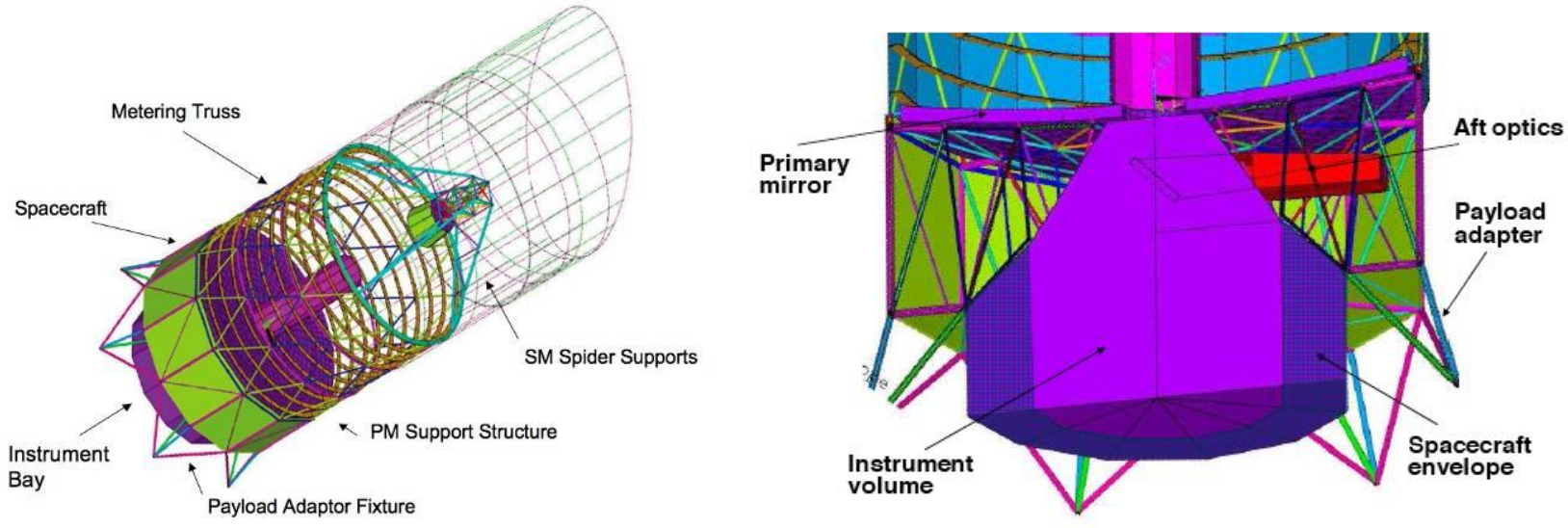

Figure 7: ATLAST-8m Observatory Structural Layout

The forward structure is similar to that of the Hubble Space Telescope. It provides the metering structure between the primary and secondary mirrors and holds the straylight baffle tube. Because of fairing length limitations, the forward structure is split into two parts. The lower structure is load carrying. It holds the secondary mirror assembly support structure and the cover doors. The upper part contains the 60 degree baffle tube which slides forward on orbit. The cover doors open and close on-orbit as required. A tripod structure extending from the primary to secondary mirror was considered but determined to be unable to achieve the desired system stiffness levels for an ultra stable telescope. A classic 4 arm secondary support structure was initially baselined with a $10.3 \mathrm{~Hz}$ first fundamental mode. But, this configuration presents diffraction challenges for internal coronagraphs. To solve this problem, a double arch secondary mirror support system was developed (Figure 8). This secondary support structure provides two $3 \times 6$ meter elliptical off-axis unobscured clear apertures for use with up to two separate coronagraphs (while retaining the full 8 meter diameter clear aperture for conventional on-axis science instruments). The first fundamental mode frequency for the double arch is $7.3 \mathrm{~Hz}$.

The back structure has multiple functions. First, it supports the primary mirror during launch. Second, the forward structure is attached to the back structure as are the spacecraft and the science instruments. A key design element of the concept is

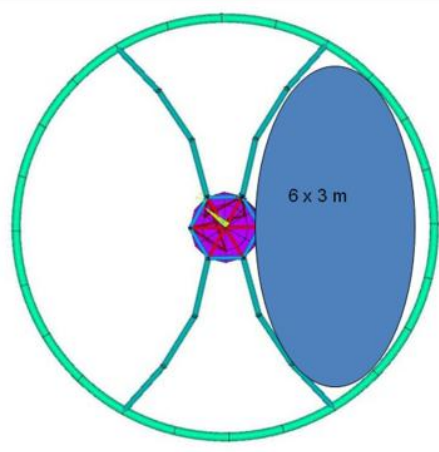

Figure 8: Double Arch Secondary Mirror support structure provides two $3 \times 6 \mathrm{~m}$ un-obscured off-axis clear apertures for Lyot stop coronagraphy with a $7.3 \mathrm{~Hz}$ first mode while preserving advantages of 8-m on-axis system. 
that all observatory mass (telescope, instruments and spacecraft) is carried through the back support structure to an interface ring which attaches via the payload adapter fixture (PAF) to the launch vehicle. This allows the use of a completely conventional spacecraft, i.e. it does not need extra mass because it does not provide the interface between the observatory and the launch vehicle.

The fundamental structural design philosophies are simplicity, modularity, and redundancy. To mitigate assembly risk, the structure is designed using a bolt-together truss structure of repeated components. Each component is fabricated with a conservative design margin and tested individually. The most challenging engineering issue is the joint interface between nearly 1300 components. The structure is designed to safely launch an 8-meter primary mirror and maintain the on-orbit optical alignment necessary to achieve a $500 \mathrm{~nm}$ diffraction limited telescope. Using fault-tolerant design principles, the PM support structure provides a 10X margin of safety by distributing the forces between 66 axial and lateral support points to keep the primary mirror launch loads at least an order of magnitude below its design limit.

Thermal: A key feature of ATLAST-8m is its extreme thermal and mechanical stability. (13) The ATLAST-8m passive thermal isolation system of MLI insulation, scarfed sun shade and straylight baffles are such that the observatory is coldbiased for all permitted observation angles relative to the sun. This allows the use of an active thermal management system to bring the observatory up to the desired $280 \mathrm{~K}$ operating temperature and to eliminate any systematic spatial or temporal thermal variations. The primary mirror temperature is actively controlled using radiative heaters applied to the back and sides. Approximately 60 independently controlled heater zones are required to minimize gradients through and across the mirror. The primary mirror axial through-thickness thermal gradient is a negligible: $0.02 \mathrm{~K}$. The secondary mirror and optical bench structure is heated using the same methodology as HST. Given the enormous thermal mass of the ATLAST$8 \mathrm{~m}$ system, it is virtually immune to transient thermal events. A 20 degree slew or 30 degree roll produces only a $0.2 \mathrm{~K}$ change in thermal gradients. The primary mirror thermal time constant is $500 \mathrm{hrs}$ to produce a $1 \mathrm{~nm}$ rms figure change.

\subsection{Spacecraft}

The spacecraft provides all pointing, power, communication, data handling, station keeping, momentum unloading, and thermal control for the ATLAST-8m telescope and its science instruments, and provides the propulsive maneuvers for midcourse corrections. (12) Key requirements include enabling the observatory to slew 60 degrees in 90 minutes (required) or 40 minutes (desired); ensuring a pointing stability of 1.6 mas; enabling the observatory to roll about the telescope's line of sight by \pm 30 degrees in 30 to 60 minutes, and provide a minimum of 4500 minutes continuous observing time before momentum unloading is required. All subsystem components are sized with a 30\% mass contingency and, when electrical power is required, a $30 \%$ reserve is included.

The spacecraft is packaged in a donut-shaped volume (Figure 9) located at the rear of the OTA, surrounding the science instrument bay. Designed for serviceability, key subsystem components are grouped into Orbital Replaceable Units (ORUs) to enable communication elements, reaction wheel assemblies, and other components to be removed and replaced during servicing. All ORUs, as well as science instruments, are removed from the rear of the spacecraft and science instrument bay. To prevent transmission of vibration into the instruments, the spacecraft volume does not physically contact the instrument bay. The spacecraft connects to the observatory structure through an active isolation system (e.g. Lockheed disturbance free payload or Northrop active strut technology). The active isolation system is part of the Attitude Control System (ACS). It isolates the science payload (OTA + science instruments) from spacecraft bus disturbances (e.g., vibrations from the reaction wheel assemblies), and provides fine pointing control for the science payload.
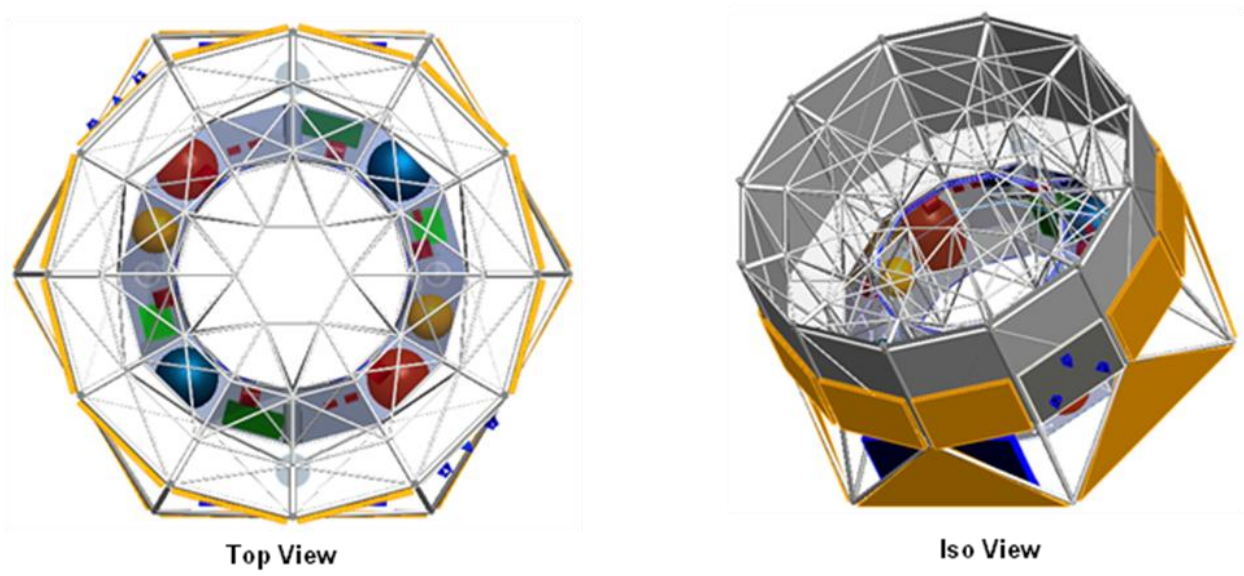

Figure 9: Modular Spacecraft Design Concept with solar panels stored inside the PAF and radiator panels and AR\&D docking interfaces mounted outside of the PAF. 
Attitude Control System (ACS): The main components of the ACS include the Fine Guidance Sensor (FGS); a coarse pointing system on the SC that includes gyros, star-trackers, reaction wheel assemblies (RWA), and/or control moment gyros (CMG), and sun sensors; a propulsion system that provides attitude control (in certain situations) and momentum unloading; Active Vibration Isolation (AVI) System between the spacecraft and observatory; and software resident on the SC Bus C\&DH system that runs the ACS algorithms. Thrusters provide the means to unload momentum periodically. The CMGs have flight heritage on Space Station and are used to achieve coarse pointing while RWs maintain that pointing for extended observation periods. The AVI system eliminates jitter to achieve sub 1.6 mas pointing stability. There are two potential approaches for the active vibration isolation system, Lockheed's disturbance free payload (Pedreiro 2003, Journal of Guidance, Control and Dynamics, Vol 26, No. 5) and Northrop's active strut technologies. The active isolation system has two roles. First, it isolates the science payload from SC bus disturbances, such as vibrations from the RWA. Second, it provides fine pointing control for the science payload, which requires a pointing stability of 1.6 mas. The FGS system has four FGS modules, two in each WFOV TMA focus (one active and one backup). ALTAST-8m controls pointing using guide stars in two FGS modules separated on the sky by 0.5 degree. This separation provides roll control about boresight at a lower bandwidth and with much better stability than the 0.2 mas rms requirement.

ATLAST-8m employs body pointing, similar to HST. The reason is driven by UV science. To maximize UV throughput, telescope pointing places the science object of interest directly onto the entrance slit of the UV spectrograph. Star Trackers command the OTA boresight pointed to within a few arc-sec of the desired target using the RWA/CMG. The active isolation system then engages using FGS feedback to minimize the apparent motion of the guide star centroid for the duration of that science exposure. During a science observation, sensors continuously monitor the travel of the AVI actuators. This information is used by the ACS software to command the RWAs, changing the orientation of the SC bus so as to maintain the AVI actuators at or near their center of travel. This feedback loop between FGS, AVI system, and RWA continues until the end of the science observation. The reaction wheels provide $698 \mathrm{~N}-\mathrm{m}$-s of momentum storage capability for a minimum of 4500 minutes continuous observation time. ATLAST- $8 \mathrm{~m}$ uses two solar panels on $10 \mathrm{~m}$ deployable booms to balance solar pressure exerted on its sunshade tube. As the observatory slews relative to the sun, the solar panel booms extend to keep the center of pressure as close as possible to the center of mass (Figure 10). Additionally, the booms have gimbal joints that articulate during observatory roll and pitch maneuvers to keep the solar panels perpendicular to the sun. Analysis shows that, with 10 meter booms extended from the spacecraft midpoint, only $35 \mathrm{~N}-\mathrm{m}$-s momentum is required for 6.25 days of continuous high-precision pointing observation. And, by making slight adjustments in boom length, indefinite observation times can theoretically be achieved.

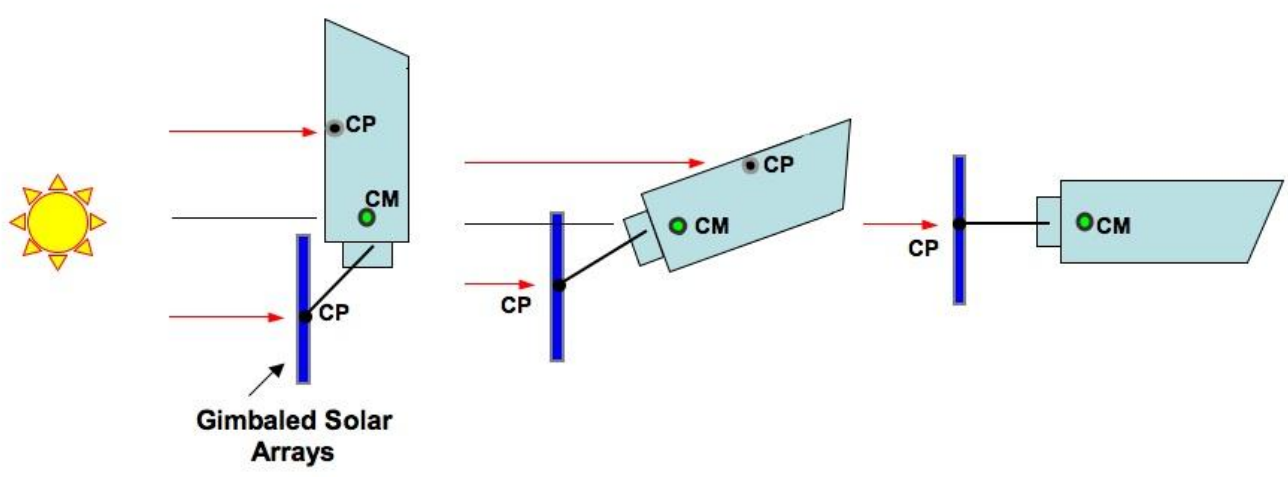

Figure 10: Solar Torque / Momentum Build-Up Mitigation Scheme for ATLAST-8m

During launch, the spacecraft is attached to the rear of the telescope structure, and does not support the observatory nor transfer launch loads to the launch vehicle (LV). Therefore, the structure is a lightweight and simple design, with aluminum tube and aluminum-lithium plate elements being the main components. Structural elements were sized for $5 \mathrm{~g}$ axial and $2 \mathrm{~g}$ lateral sustained loads, with a yield and ultimate factors of safety of 1.25 and 1.4, respectively. After separating from the LV, the Solar Arrays (SA) and the steerable High Gain Antenna (HGA) are deployed.

Thermal Control System (TCS): The TCS provides thermal control for the spacecraft and science instruments, including the OTA primary mirror. Radiators $\left(30 \mathrm{~m}^{2}\right)$ are mounted around the circumference of the OTA and spacecraft to dissipate the $11,160 \mathrm{~W}$ of heat load from the spacecraft components and science instruments. Of the $2400 \mathrm{~W}$ required to maintain the primary mirror at $280 \mathrm{~K}, 2000 \mathrm{~W}$ is scavenged from the science instruments via cold plates, although the TCS is designed with $1600 \mathrm{~W}$ of backup in case the science instruments are powered down or are being serviced. The spacecraft is wrapped in 50-layer MLI blankets, helping to maintain the spacecraft at an operational steady state sink temperature of $300 \mathrm{~K}$ $( \pm 10 \mathrm{~K})$. The main functions of the TCS are to dissipate the heat from the power consumed by the SC and to keep the SC Bus components in their operational temperature range. 


\subsection{Mass and Power Budgets}

Master Equipment Lists (MELs) were generated for each component of the optical telescope assembly, instruments and spacecraft. These MELs were used to estimate the mass of the payload and to size the power system (Table 1). In table 1, the primary mirror is allocated $19,250 \mathrm{~kg}$ for a $155 \mathrm{~mm}$ thick ULE mirror. With a $30 \%$ contingency, the $\mathrm{PM}$ could be $25,000 \mathrm{~kg}$. The existing Schott Zerodur blank is close to $300 \mathrm{~mm}$ thick and has a $28 \mathrm{~m}$ radius of curvature. Changing its radius to $24 \mathrm{~m}$ and removing top and bottom surface damage yields a $175 \mathrm{~mm}$ thickness. If a $175 \mathrm{~mm}$ thick Zerodur mirror is used, its mass would be $25,000 \mathrm{~kg}$ and the total payload mass would be $50,270 \mathrm{~kg}$. But, since the mirror cannot get more massive, the payload mass margin against the Ares V 65,000 capacity is $29 \%$ (60\% excluding the mirror). If a $175 \mathrm{~mm}$ thick ULE mirror is used, its mass would be $21,750 \mathrm{~kg}$ and the payload mass margin would be $38 \%$. For additional conservatism, the ATLAST-8m OTA structure (primary mirror support structure, launch locks and payload adapter fixture) is 'sized' based on a 25,000 kg primary mirror.

\begin{tabular}{|l|c|c|}
\hline \multicolumn{3}{|c|}{ Table 1: ATLAST-8m Mass and Power Budget } \\
\hline \multicolumn{1}{|c|}{ Parameter } & Value & Units \\
\hline Mass & & \\
\hline Optical Telescope Assembly & 33,908 & $\mathrm{~kg}$ \\
\hline Primary Mirror Assembly & 24,988 & $\mathrm{~kg}$ \\
\hline Primary Mirror & 19,250 & $\mathrm{~kg}$ \\
\hline Primary Mirror Mount & 5,738 & $\mathrm{~kg}$ \\
\hline Secondary Mirror and Aft-Optics Assemblies & 2,118 & $\mathrm{~kg}$ \\
\hline Structure \& Instrument Bay & 5,360 & $\mathrm{~kg}$ \\
\hline Thermal & 1,442 & $\mathrm{~kg}$ \\
\hline Instruments & 1,789 & $\mathrm{~kg}$ \\
\hline Spacecraft & 4,157 & $\mathrm{~kg}$ \\
\hline Propellant & 4,666 & $\mathrm{~kg}$ \\
\hline TOTAL PAYLOAD & $\mathbf{4 4 , 5 2 0}$ & $\mathbf{k g}$ \\
\hline Launch Vehicle Payload Capacity & 65,000 & $\mathrm{~kg}$ \\
\hline Launch Vehicle Mass Margin (\%) & 46 & $\%$ \\
\hline Payload Mass if Primary Mirror = 25,000 kg & 50270 & $\mathrm{~kg}$ \\
\hline Launch Vehicle Mass Margin & 15,270 & $\mathrm{~kg}$ \\
\hline Payload Mass without Primary Mirror & 25,270 & $\mathrm{~kg}$ \\
\hline Payload Mass Margin (\%) & 60 & $\%$ \\
\hline Spacecraft Bus Power Requirement & 8,589 & $\mathrm{~W}$ \\
\hline Projected End of Life Solar Panel Production & 11,200 & $\mathrm{~W}$ \\
\hline Solar Panel Power Margin (\%) & 30 & $\%$ \\
\hline
\end{tabular}

\section{CONCLUSION}

The ATLAST Astrophysics Concept Study has developed a detailed point design for an 8-meter monolithic aperture UV/OIR space telescope called ATLAST-8m. The mission concept is specifically designed to take advantage of the unprecedented mass and volume capabilities of a potential NASA heavy lift launch vehicle. The key fundamental design paradigm for ATLAST-8m is simplicity. Simple high TRL technology offers lower cost and risk. The capacities of potential heavy lift launch vehicles allow one to use mass to buy down performance, cost and schedule risk. An 8-meter class UV/optical space observatory with its very high angular resolution, very high sensitivity, broad spectral coverage, and high performance stability offers the opportunity to answer some of the most compelling science questions. How did the present Universe come into existence and of what is it made? What are the fundamental components that govern the formation of today's galaxies? How does the Solar System work? What are the conditions for planet formation and the emergence of life? And maybe most importantly, are we alone?

\section{ACKNOWLEDGEMENTS}

The authors wish to acknowledge the contributions of David Jones, Dan Thomas, Sharon Fincher, Scott Thomas, of NASA MSFC, Pete Capizzo of Raytheon, and Kevin Thompson of Jacobs, for spacecraft design and configuration; and Jessica Garcia of Jacobs and Eric Waters of Dynamics Concepts, Inc. for Ares V performance analysis. 


\section{REFERENCES}

1. Stahl, H. Philip, Marc Postman, William R. Arnold Sr., Randall Hopkins, Linda Hornsby, Gary E. Mosier and Bert A. Pasquale, "Design for an 8 meter monolithic UV/OIR space telescope", SPIE Proceedings 7436, 2009.

2. Stahl, H. Philip, “Ares V launch capability enables future space telescopes”, SPIE Proc.6687, 66870L (2007)

3. Stahl, H. Philip, Phil Sumrall, and Randall Hopkins, “Ares V launch vehicle: an enabling capability for future space science missions", Acta Astronautica, Elsevier Ltd., 2009, doi:10.1016/j.actaastro.2008.12.017

4. ATLAST Astrophysics Mission Concept Study final report is available at: http://www.stsci.edu/institute/atlast

5. Postman, Marc, et. al., "A Large Monolithic-Aperture Optical/UV Serviceable Space Telescope Deployed to L2 by an Ares-V Cargo Launch Vehicle”, Science Associated with Lunar Exploration Architecture, Tempe, AZ, 28 Feb 2007

6. Stahl, "Ares V an Enabling Capability for Future Space Science Missions", Science Associated with Lunar Exploration Architecture, Tempe, AZ, 28 Feb 2007.

7. Hopkins, Randall and H. Philip Stahl, "A large monolithic telescope placed at the second Sun-Earth Lagrange point”, AIAA Space 2007, AIAA-2007-6166, 2007

8. Stahl, H. Philip and Marc Postman, "8 meter Monolithic UV/Optical Space Telescope", 59th International Astronautical Congress, Glasgow, Scotland, Sept 2008.

9. Stahl, H. Philip, "Design study of an 8 meter monolithic mirror uv/optical space telescope", SPIE Proc. 7010, (2008)

10. Pasquale, Bert A., H. Philip Stahl, Joseph M. Howard, David L. Aronstein, Lee D. Feinberg and Qian Gong, "Comparative Concepts for ATLAST Optical Designs", SPIE Proceedings 7731, 2010.

11. Arnold, William R., and H. Philip Stahl, "Structural design considerations for an 8-m space telescope", SPIE Proceedings 7425, 2009.

12. Hopkins, Randall C., Peter Capizzo, Sharon Fincher, Linda S. Hornsby, David Jones, Gary Mosier, H. Philip Stahl, Dan Thomas and Kevin S. Thompson, "Spacecraft conceptual design for the 8-meter Advanced Technology Large Aperture Space Telescope (ATLAST)", SPIE Proceedings 7731, 2010.

13. Hornsby, Linda, Randall C. Hopkins, H. Philip Stahl, “Thermal analysis of the Advanced Technology Large Aperture Space Telescope (ATLAST) 8 meter primary mirror”, SPIE Proceedings 7731, 2010

14. Postman, Marc, et. al., "Science with an 8-meter to 16-meter optical/UV space telescope", SPIE Proc.7010, (2008)

15. Postman, Marc, Wesley A. Traub, William R. Oegerle, Thomas M. Brown, H. Philip Stahl, Daniella Calzetti, Dennis C. Ebbets, Ronald S. Polidan, "Science flowdown requirements for ATLAST: implications for technology development and synergies with other future facilities“, SPIE Proceedings 7731, 2010.

16. Stahl, H. Philip, Kyle Stephens, Todd Henrichs, Christian Smart, and Frank A. Prince, "Single Variable Parametric Cost Models for Space Telescopes”, Optical Engineering Vol.49, No.06, 2010 


\title{
ATLAST-8 Mission concept study for 8-meter Monolithic UV/Optical Space Telescope
}

\author{
H. Philip Stahl, NASA MSFC \\ Marc Postman, STScI \\ William R. Arnold, Sr., Jacobs ESTS Group, MSFC \\ Randall Hopkins, NASA MSFC \\ Linda Hornsby, Jacobs ESTS Group, MSFC \\ Gary E. Mosier, NASA GSFC \\ Bert A. Pasquale, NASA GSFC
}




\section{History}

Marc Postman of STScI, myself and the MSFC Advanced

Concepts Group Spacecraft Study Team lead by Randall Hopkins have been working on massive 6 to 8 meter class space telescopes since early 2007.

Postman, "A Large Monolithic-Aperture Optical/UV Serviceable Space Telescope Deployed to L2 by an Ares-V Cargo Launch Vehicle", Science Associated with Lunar Exploration Architecture, Tempe, AZ Feb. 28, 2007

Stahl, "Ares V an Enabling Capability for Future Space Science Missions", Science Associated with Lunar Exploration Architecture, Tempe, AZ Feb. 28, 2007

Hopkins and Stahl, "A large monolithic telescope placed at the second SunEarth Lagrange point”, AIAA Space 2007, AIAA-2007-6166, 2007

Which lead to the 2008 Multi-Institutional ATLAST Astrophysics Mission Concept Study whose final report is available at: http://www.stsci.edu/institute/atlast 


\section{ATLAST}

The Astrophysics Mission Concept Study Advanced Technology Large-Aperture Space Telescope (ATLAST) examined 3 different mission concepts:

ATLAST-8m (8-meter monolithic aperture)

ATLAST-9m (9.2 meter segmented aperture)

ATLAST-16m (16.8 meter segmented aperture)

All ATLAST concepts are UV/OIR (110 nm to $2400 \mathrm{~nm}$ )

ATLAST-8m will have exceptional performance capability:

- 16 milli-arc-sec (mas) angular resolution

- $\sim 500 \mathrm{~nm}$ diffraction limit

- $\sim 50 \mathrm{~m}^{2}$ collecting aperture

- 500 hour thermal time constant 


\section{Science}

The science case for ATLAST is compelling and documented:

Postman, et. al., "Science flowdown requirements for ATLAST:

implications for technology development and synergies with other future facilities “, SPIE Proceedings 7731, 2010.

Postman, Marc, et. al., "Science with an 8-meter to 16-meter optical/UV space telescope", SPIE Proc. 7010, 2008.

ATLAST-8m has the performance necessary to

detect the potentially rare occurrence of life.

reveal underlying physics driving star formation,

trace the complex interactions between dark matter, galaxies, and the intergalactic medium 


\section{ATLAST-8 is fully Documented}

ATLAST Astrophysics Mission Concept Study:

http://www.stsci.edu/institute/atlast/index_html_ATLASTMissionConceptStud

\section{y_Page}

Hopkins, et. al., "Spacecraft conceptual design for the 8-meter Advanced

Technology Large Aperture Space Telescope (ATLAST)”, SPIE 7731. 2010.

Hornsby, et. al., "Thermal analysis of the Advanced Technology Large Aperture

Space Telescope (ATLAST) 8 meter primary mirror”, SPIE 7731. 2010.

Pasquale, et. al., "Comparative Concepts for ATLAST Optical Designs", $\underline{\text { SPIE }}$

7731. 2010.

Arnold and Stahl, "Structural design considerations for an 8-m space telescope", SPIE 7425, 2009.

Stahl, et. al. "Design for an 8 meter monolithic UV/OIR space telescope", $\underline{\text { SPIE }}$ 7436, 2009.

Stahl, "Design study of an 8 meter monolithic mirror uv/optical space telescope",

SPIE Astronomy Conference, Marseille, France, June 2008 
ATLAST-8 requires a heavy lift launch vehicle with capabilities similar to Ares $\mathrm{V}$ :

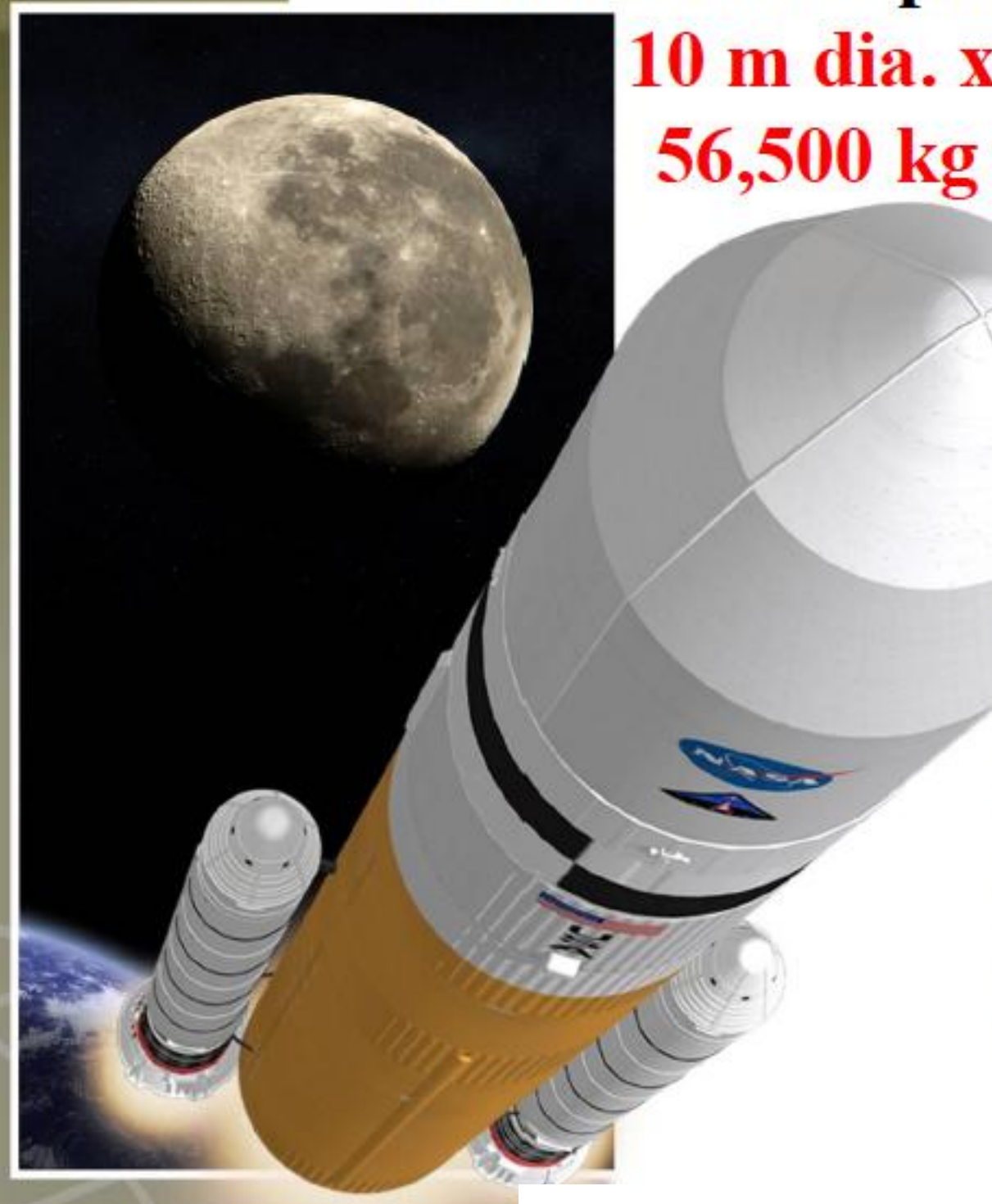

While constellation is currently in a state of flux, MSFC is currently studying several heavy lift vehicle options, many of which could launch ATLAST-8m.

LV 51.00.48 configuration used for ATLAST study. 


\section{Mission Concept - Launch Configuration}

Fundamental idea is to trade Mass for Simplicity to increase Performance and Reduce Risk.

ATLAST-8m is specifically designed to fully utilize the Ares V payload mass and volume capacities.

$8.8 \mathrm{~m}$ dia. dynamic envelop allows:

$8 \mathrm{~m}$ diameter telescope

$17 \mathrm{~m}$ tall dynamic envelop allows:

SM to be launched fully deployed.

$65 \mathrm{mt}$ payload mass allows:

solid meniscus glass mirror

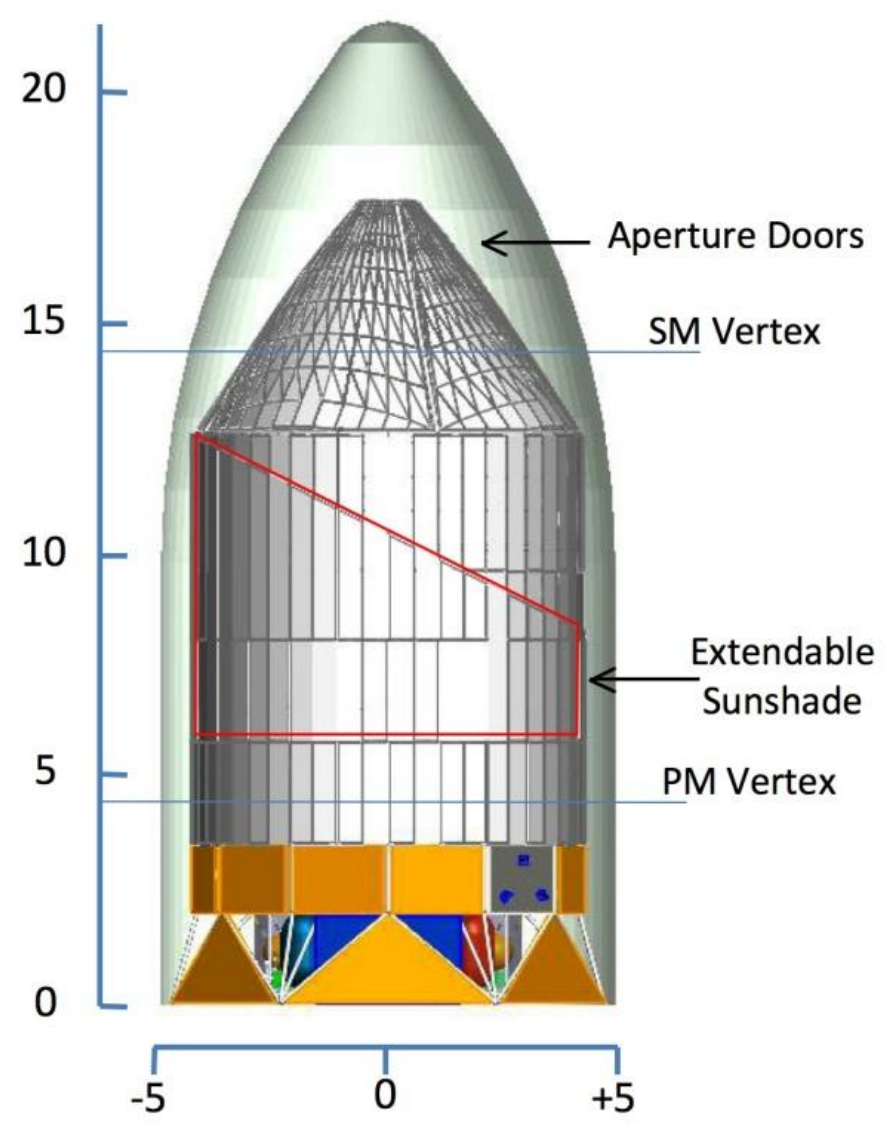




\section{ATLAST 8-meter in Ogive Shroud}

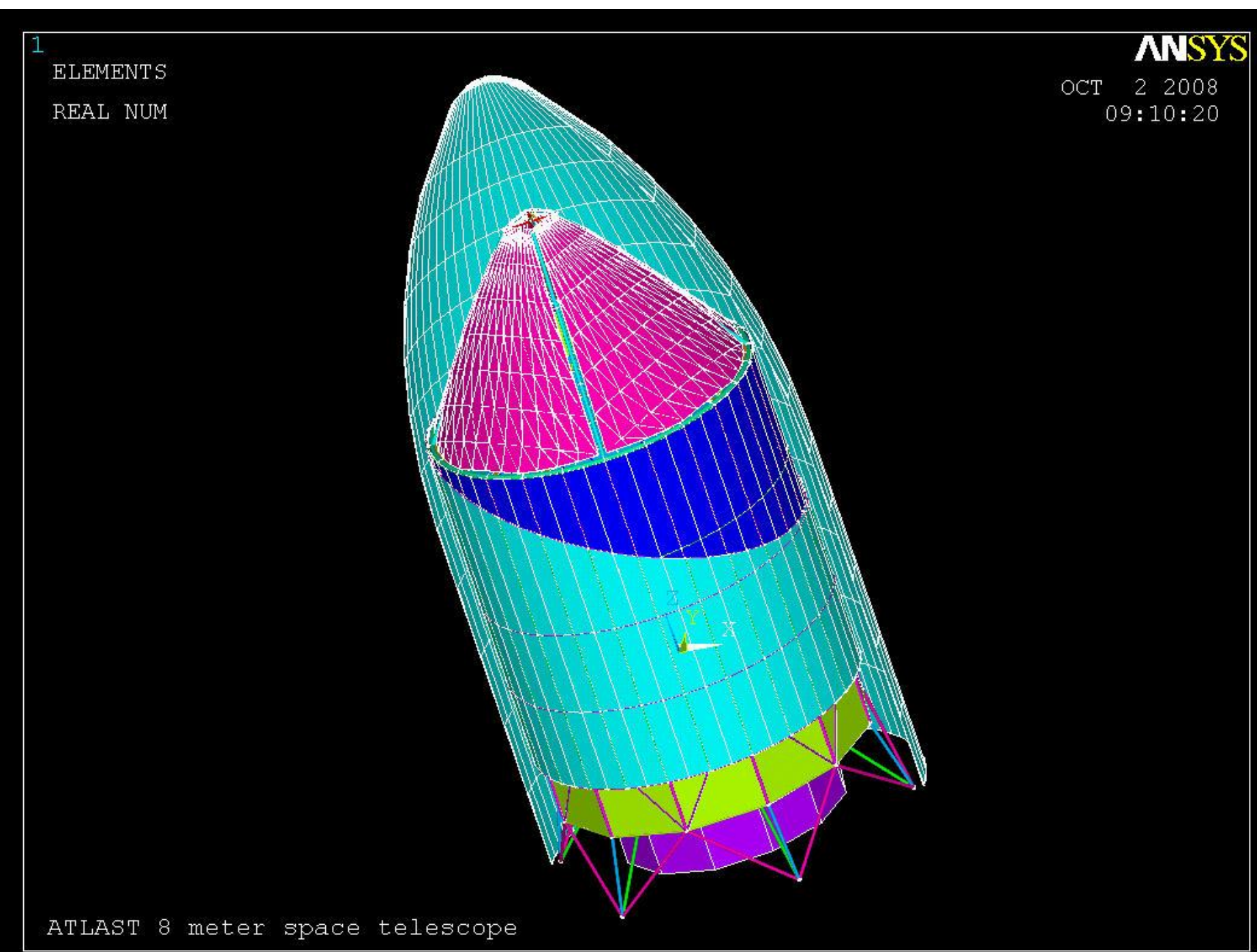




\section{Doors Open - view of light baffles}

\section{open doors before or after deploying tube}

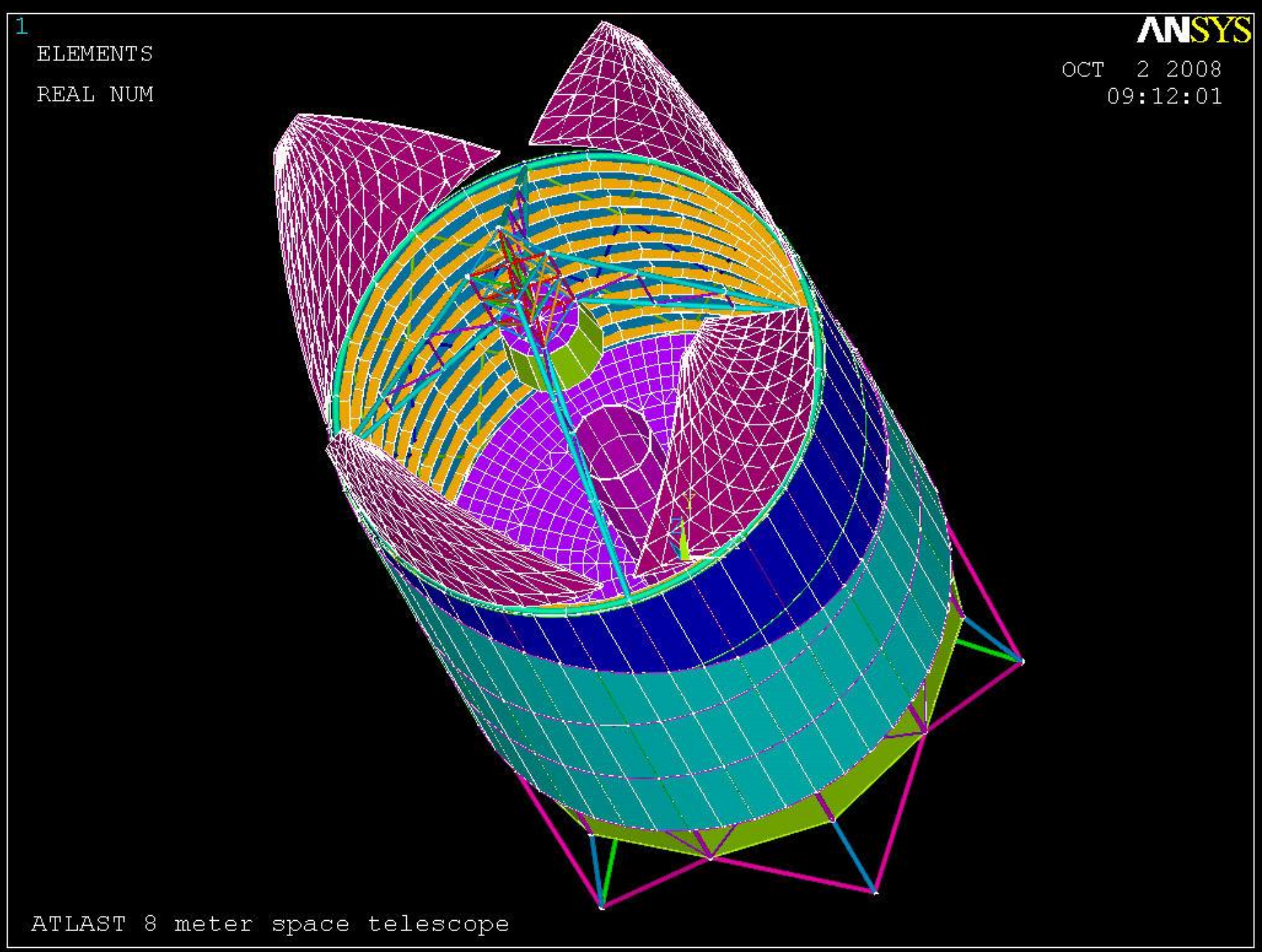




\section{Observatory with 60 deg Light Tube}

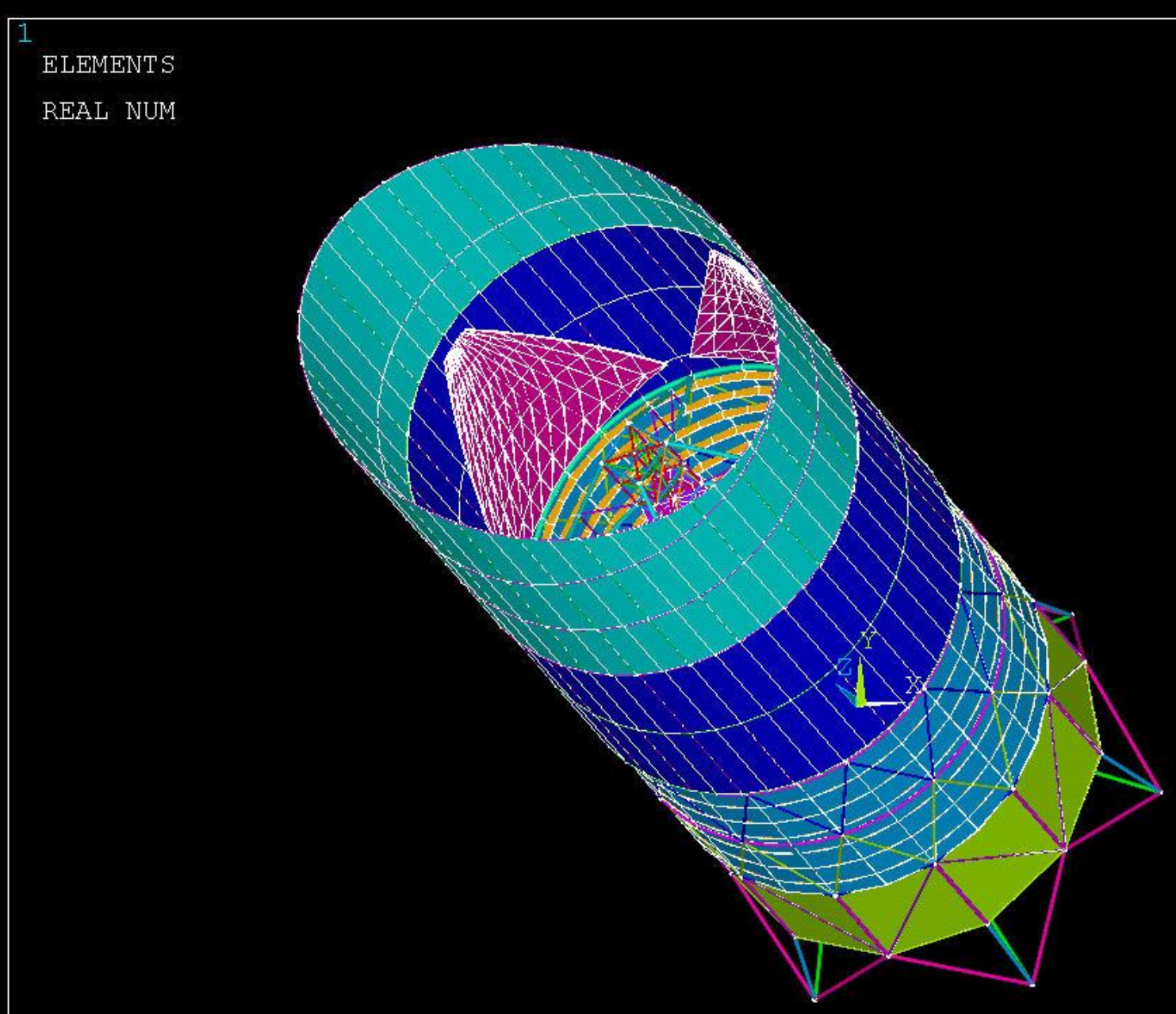




\section{Mission Concept - On-Orbit}

Once on orbit, the protective doors open, the 60 degree scarfed sunshade slides forward and the solar panels deploy

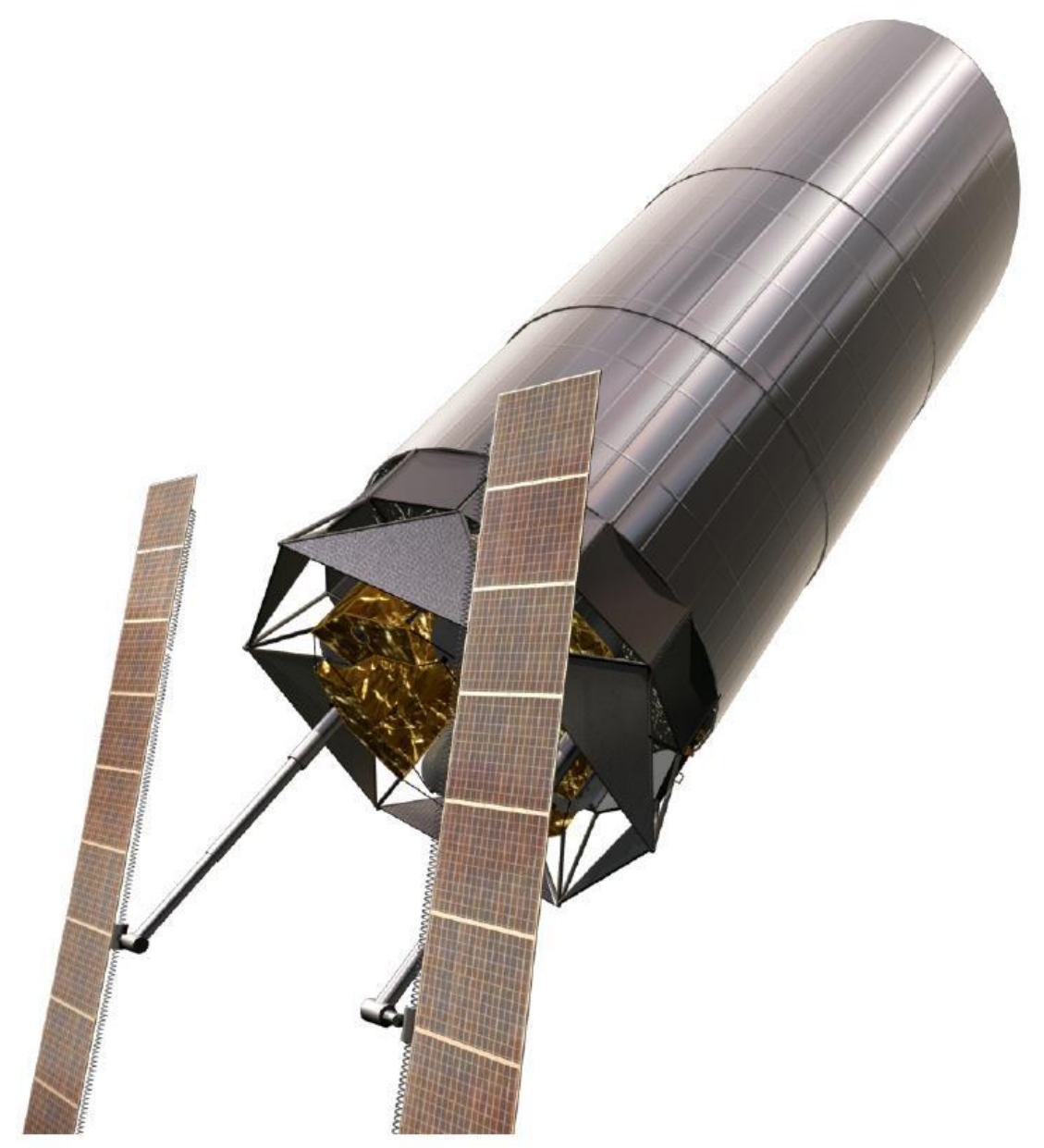




\section{Dual-Field Optical Design with Three Foci}

On-Axis Cassegrain and Two off-axis TMA Foci

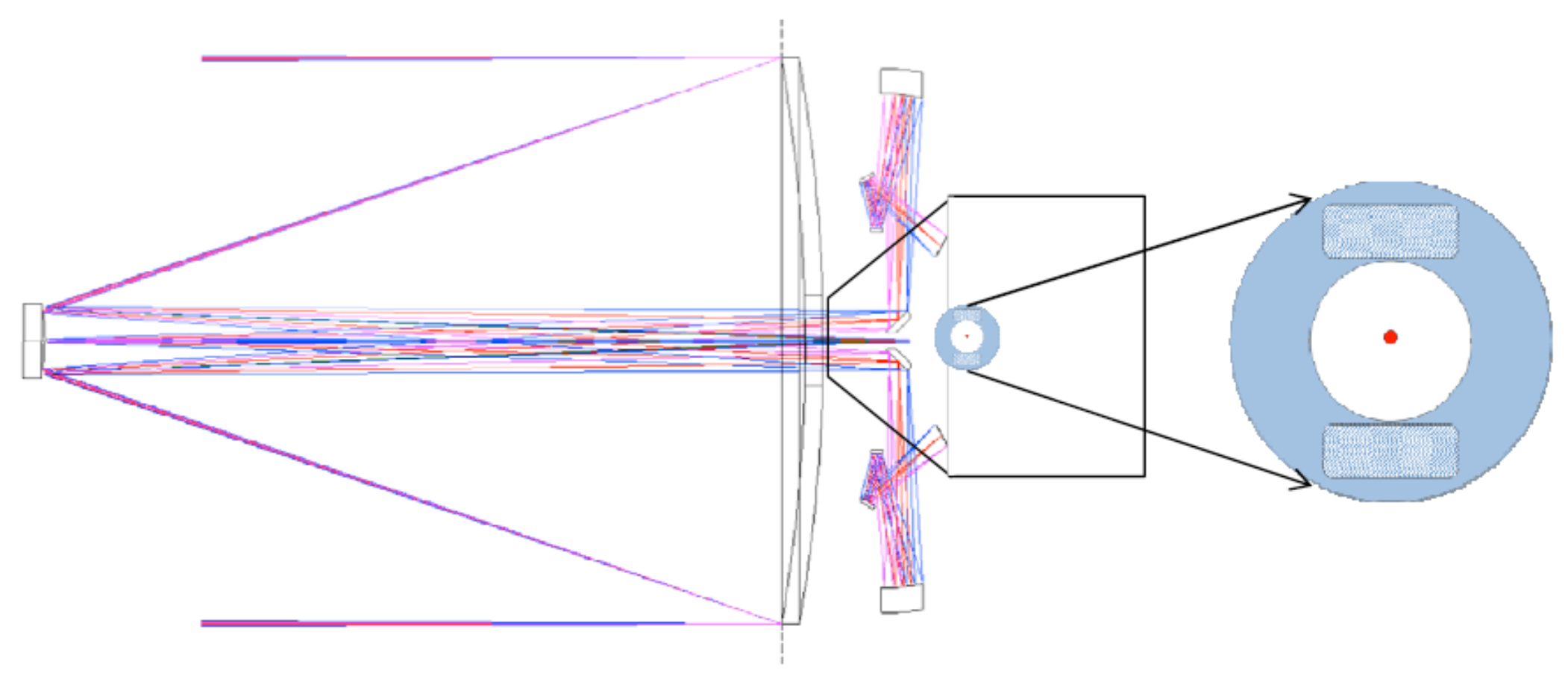

all feed $4 \mathrm{~m}$ diameter $\mathrm{x} 4.5 \mathrm{~m}$ deep instrument bay.

Plate-scale is $13 \mathrm{mas} / 10 \mu \mathrm{m}$ 


\section{Notional Science Instruments}

Cassegrain Focus could have 2 instruments (exoplanet \& UV spectrometer) and 2 WFS.

TMA Focus could have 3 instruments (WFOV Imager, Multi-

Object Spectrograph and Integral Field Unit spectrograph) and 2 FGS and 2 WFS.

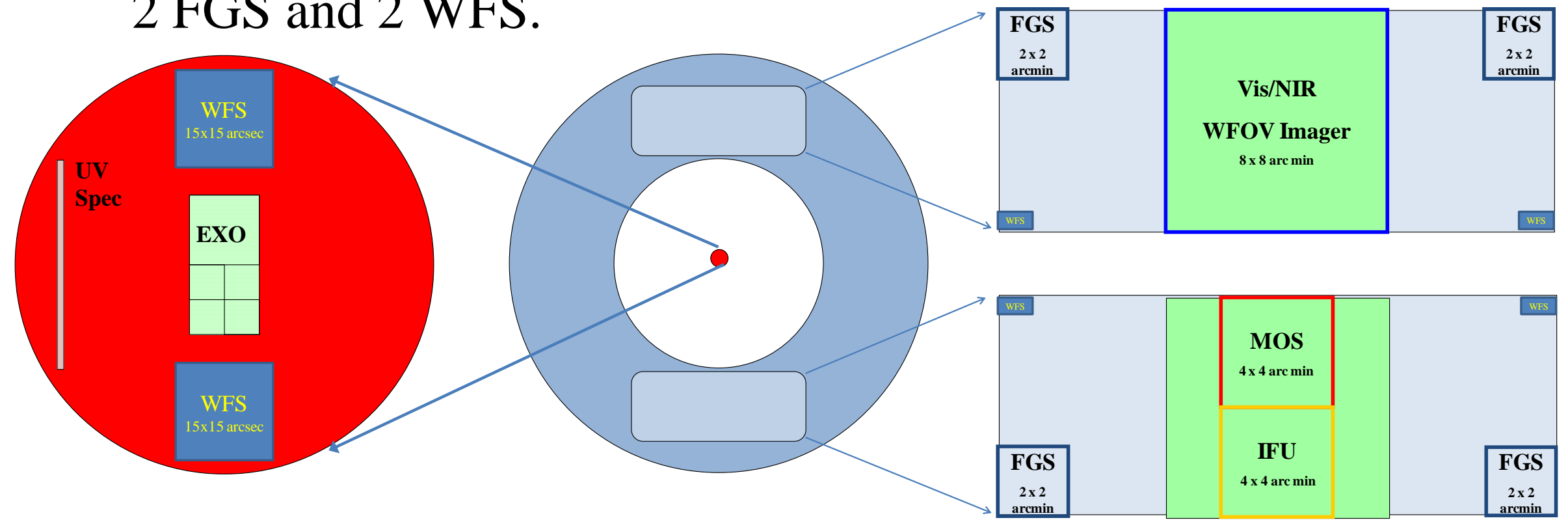

Actual instruments will be based on a peer-reviewed competition, driven by the most compelling science. 


\section{Solid Meniscus Primary Mirror}

Ares V's mass capacity allows us to reduce risk and cost by using a solid meniscus glass mirror.

Such mirrors are extremely thermally stable \& mechanically stiff.

A solid meniscus mirror also allows one to cost effectively achieve a very smooth optical surface, a mirror property that is important for ultra-high contrast imaging.

Infrastructure exists to make 8-m mirror blanks and fabricate them to $<10 \mathrm{~nm}$ rms. 


\section{Primary Mirror Support Structure}

Primary Mirror must survive launch and perform on orbit.

Mounts which work well on Earth do not work well in Space.

Design study resulted in a conventional truss structure mount.

PM structure provides 10X safety margin during launch via a combination of 66 axial launch locks as well as radius and tangential supports.

Key is to minimize bending.
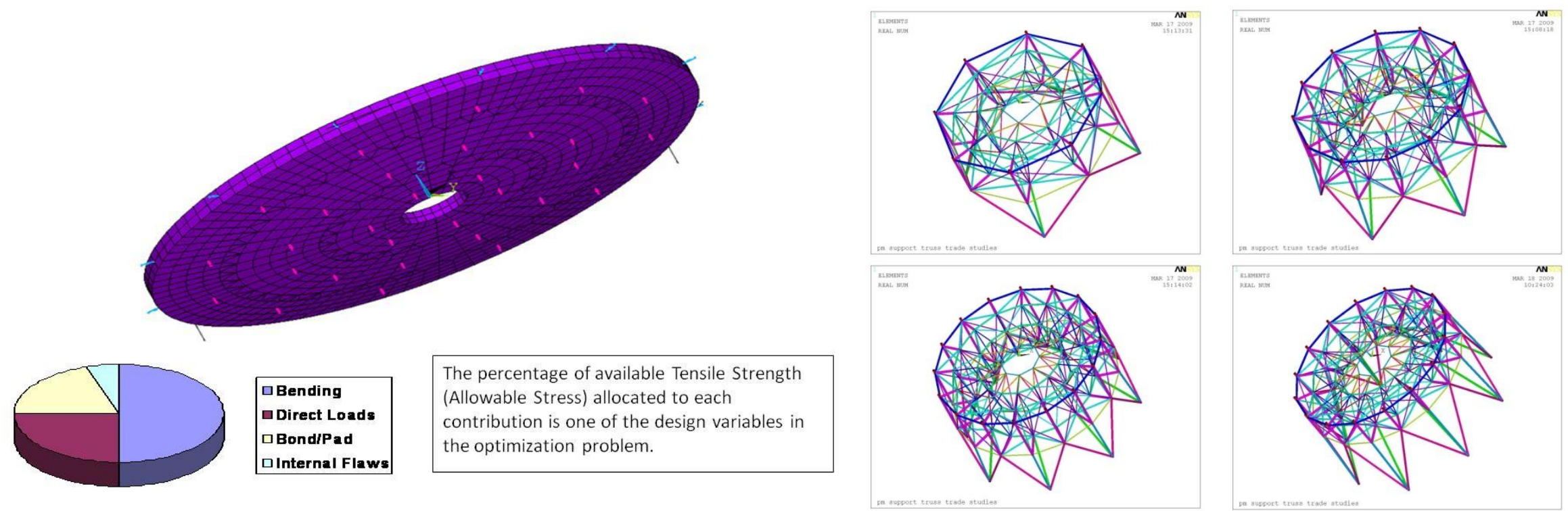


\section{Secondary Mirror Support Structure}

Similar design trade studies were performed for SM Structure.

Key criteria is fundamental mode frequency for Launch Survival and on-orbit Pointing Stability

4 Spiders $\quad 10.3 \mathrm{~Hz}$

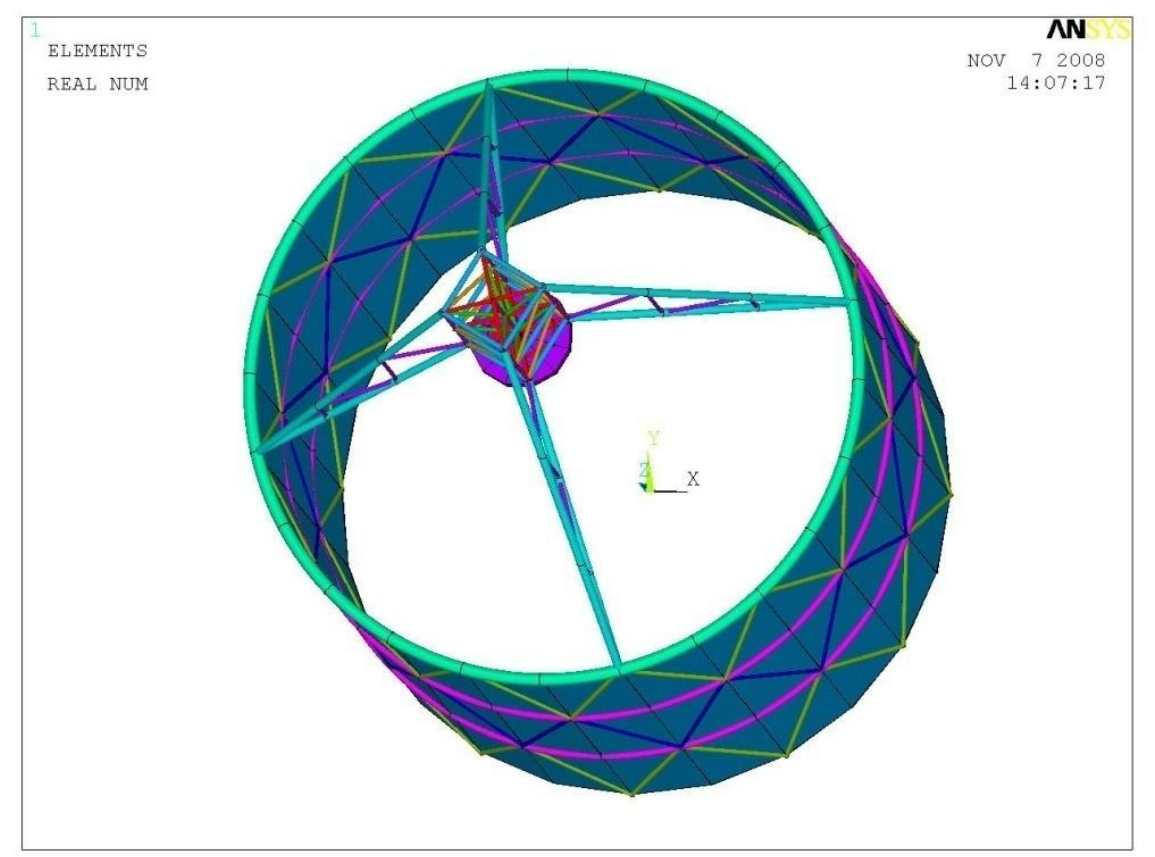

3 Spiders

$9.5 \mathrm{~Hz}$

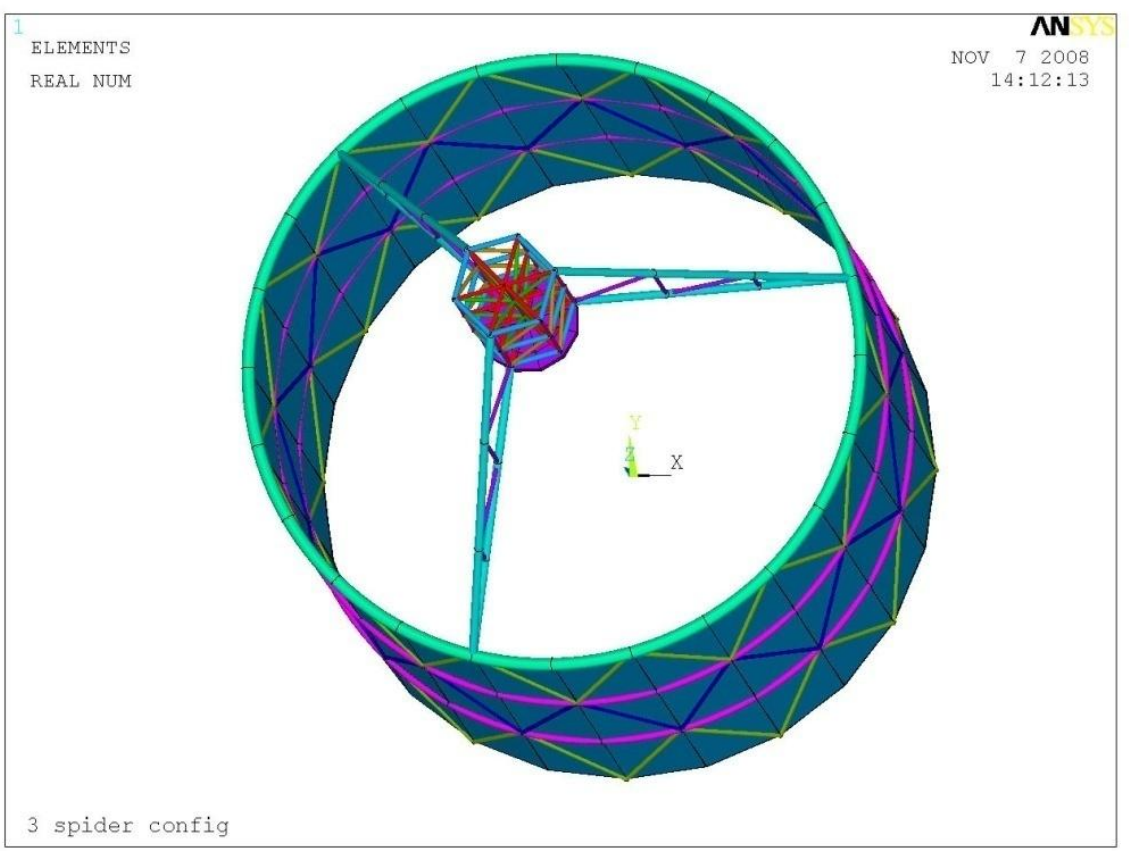




\section{Baseline - 4 Spiders 10.3 Hz First Mode}

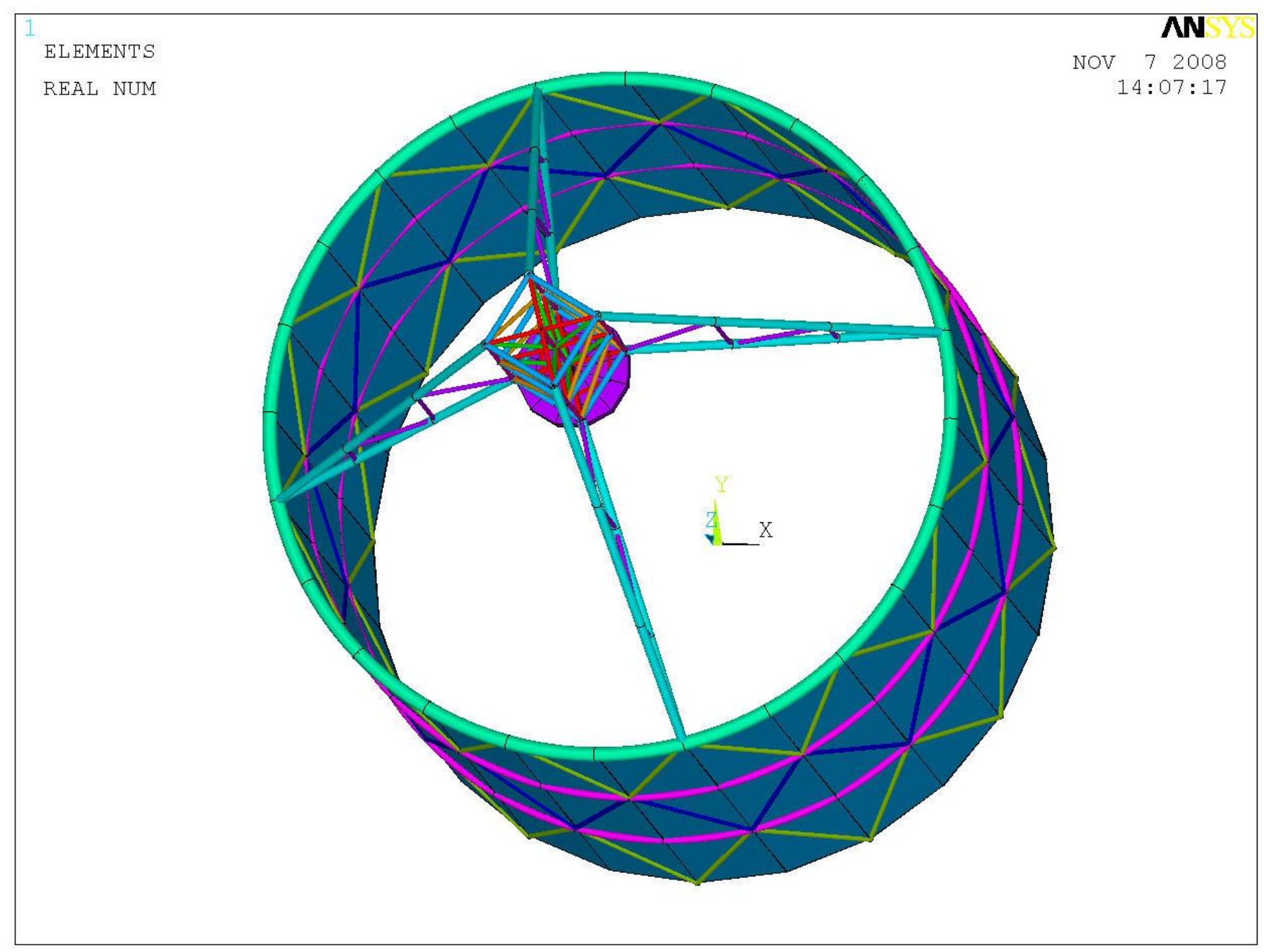




\section{Spiders - 9.5 Hz First Mode}

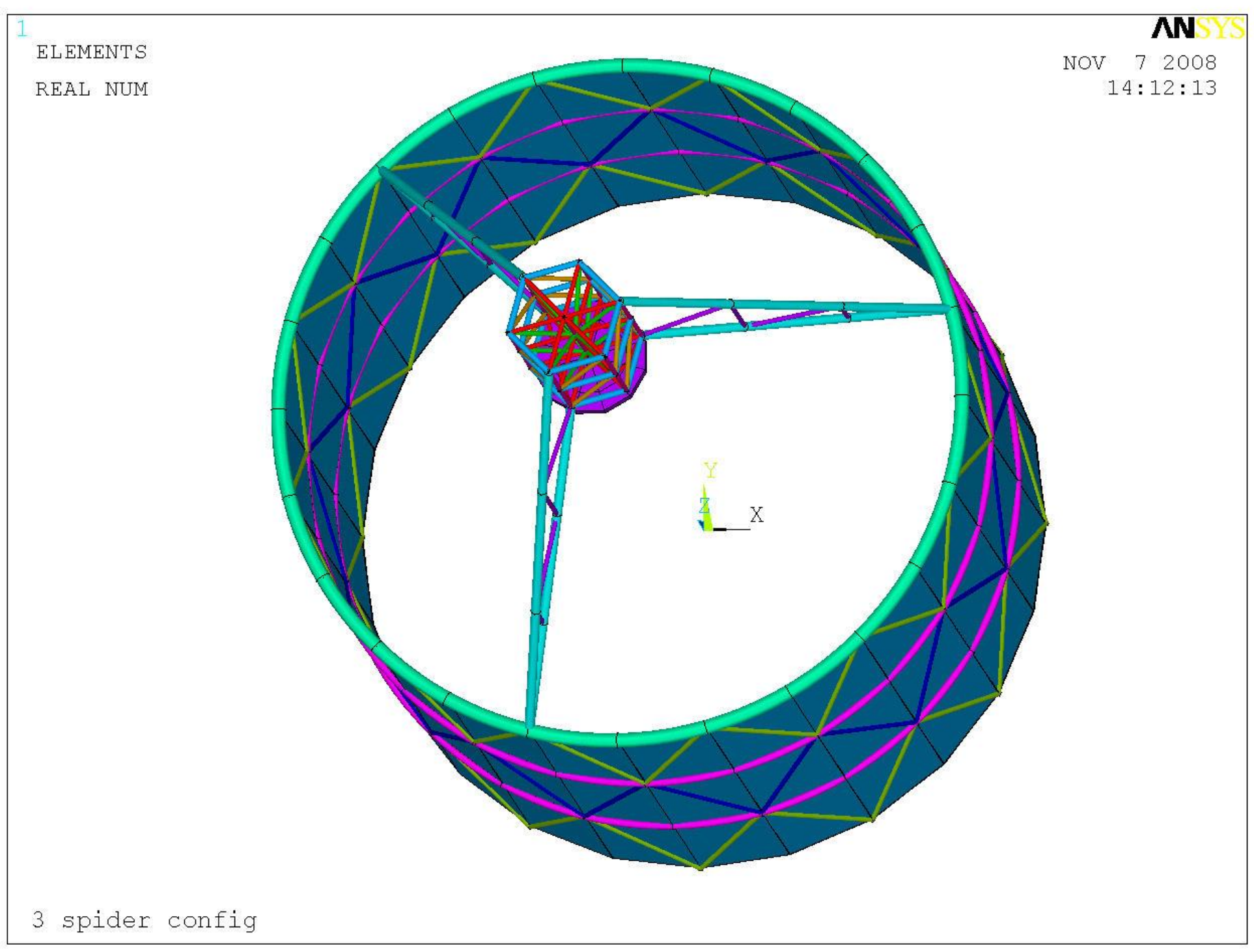




\section{Off-Axis Dual Focus Design Concept}

For high-contrast imaging of exo-planets, Internal Coronagraphs desire un-obscured aperture to avoid diffraction effects.

Off-axis systems are hard to make and align to high precision.

Off-axis systems are not compact.

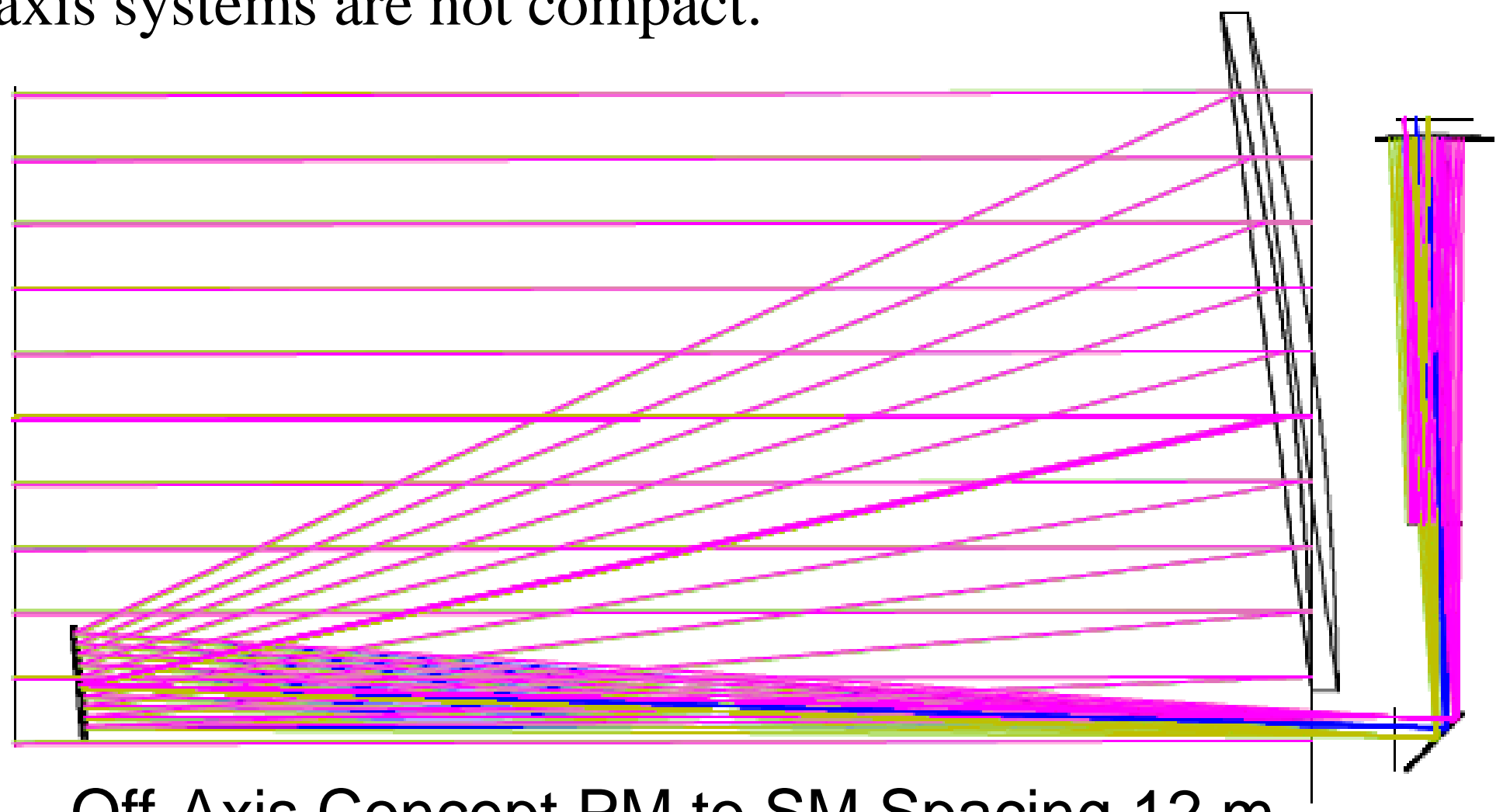

Off-Axis Concept PM to SM Spacing $12 \mathrm{~m}$ 


\section{Deployed Off-Axis Secondary Mirror}

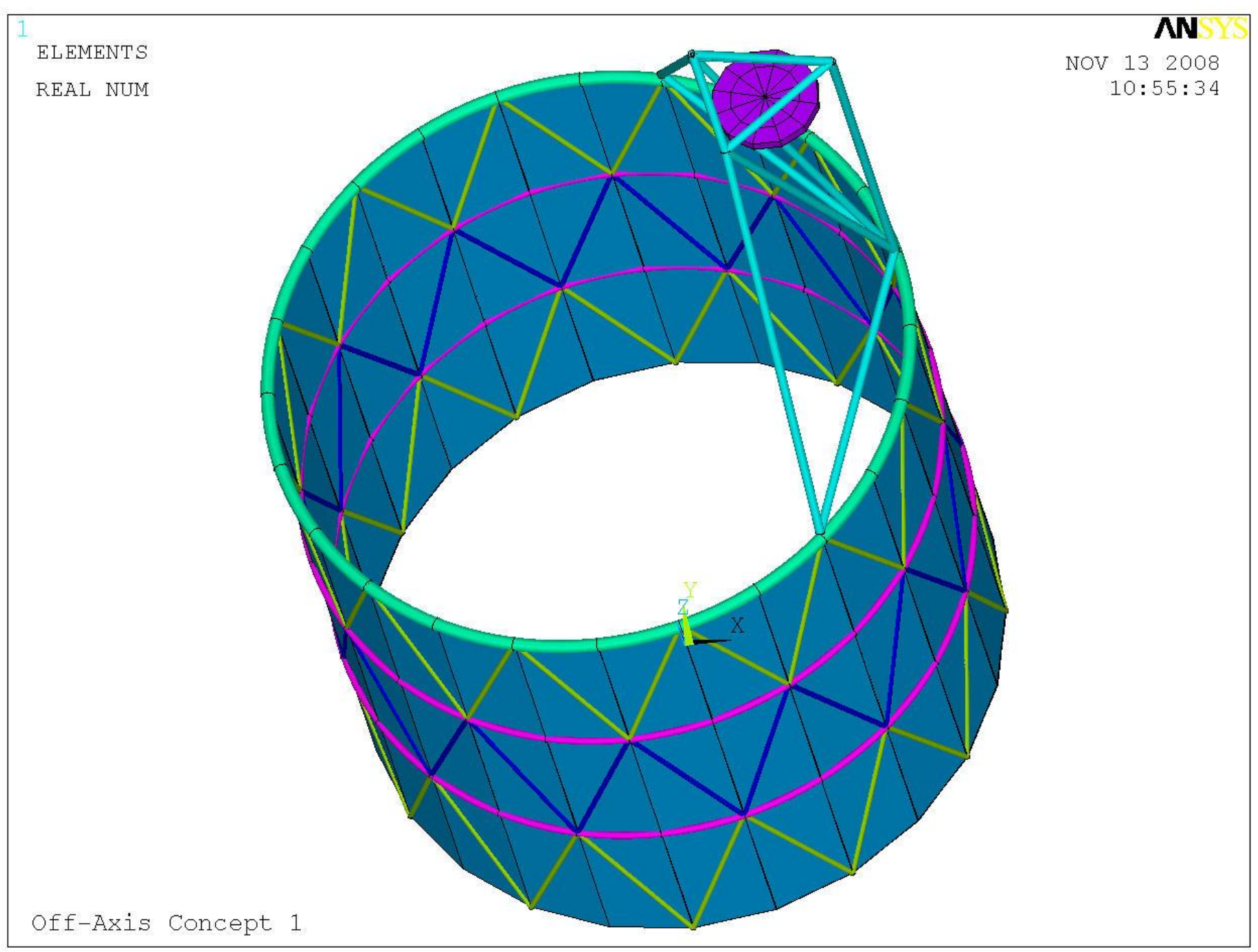




\section{Stowed Off-Axis Secondary Mirror}

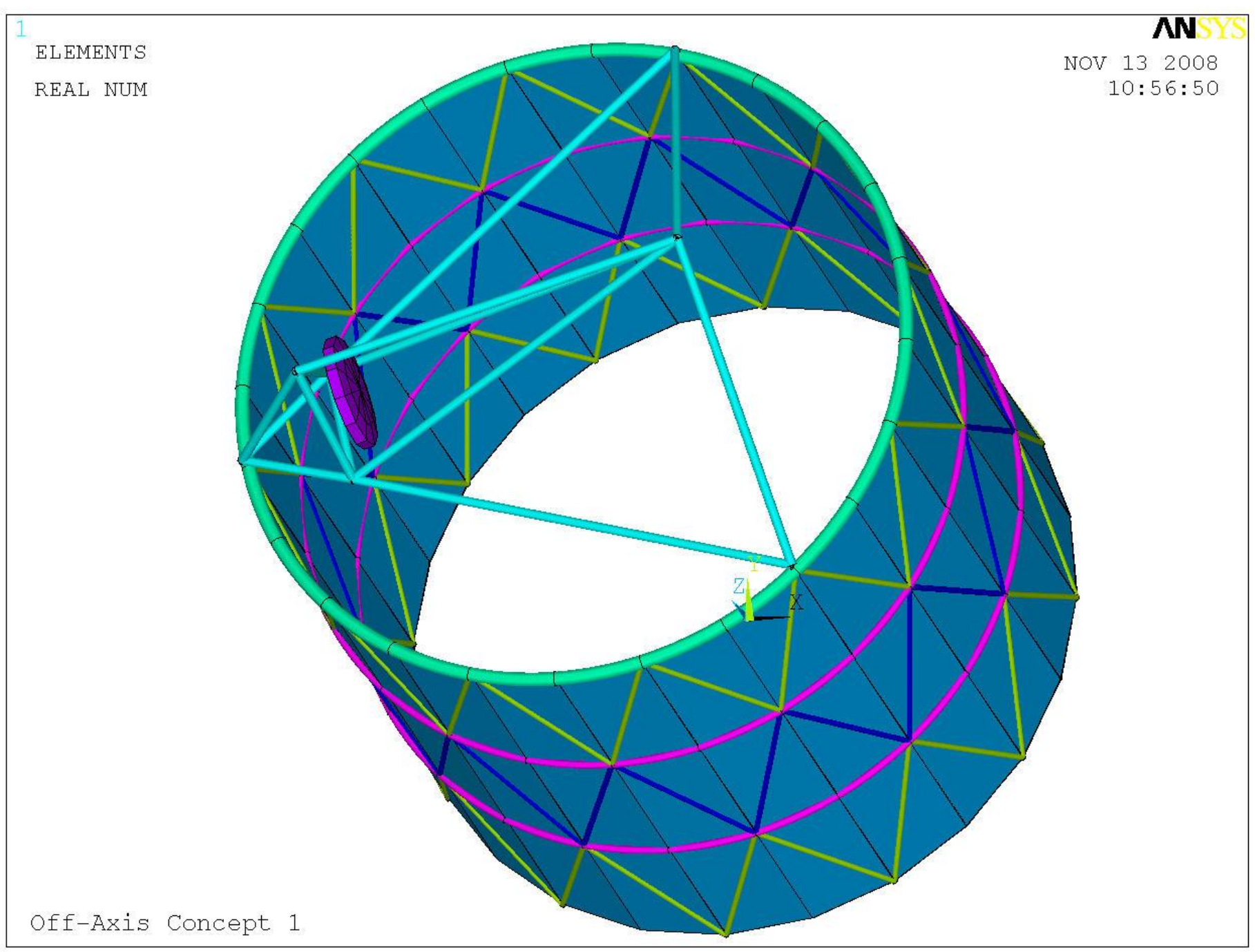




\section{Double Arch Spider Off-Axis Sub-Aperture of On-Axis Design}

On-Axis Mirrors can be made 'smoother' than Off-Axis.

Double Arch SM Support:

Has Two Off-Axis UnObscured Apertures

Can put a Coronagraph behind Each

Or, can combine Apertures.

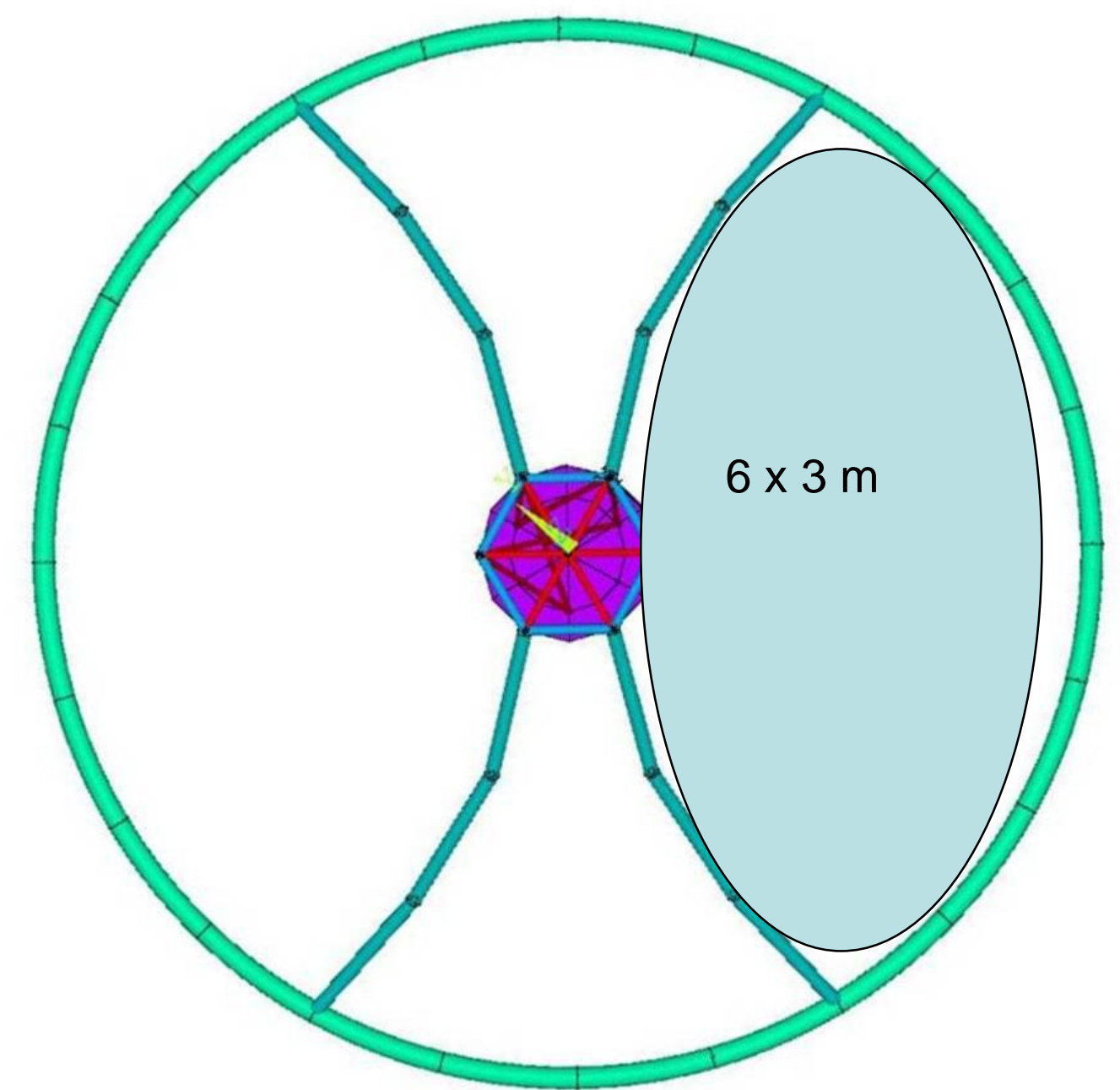




$$
\theta
$$




\section{ATLAST-8m is extremely thermally stable}

Passive Thermal Control cold biases observatory $<140 \mathrm{~K}$

50 layers of MLI \& 60 deg scarfed sun-shade

Active Thermal Control keeps observatory at $280 \mathrm{~K}+/-0.1 \mathrm{~K}$

Heaters on the PM, SM, metering truss and spiders

Primary Mirror is extremely Thermally Stable

Primary mirror axial through-thickness thermal gradient is $\mathbf{0 . 0 2 K}$

20 degree slew or 30 degree roll produces only a $\mathbf{0 . 2 K}$ change in thermal gradients.

PM thermal time constant is $500 \mathrm{hrs}$ to produce a $1 \mathbf{~ n m ~ r m s ~ f i g u r e ~}$ change. 


\section{Passive Cold Bias $<140 K$}

Analyzed PM

Temperature as a function of sun angle.

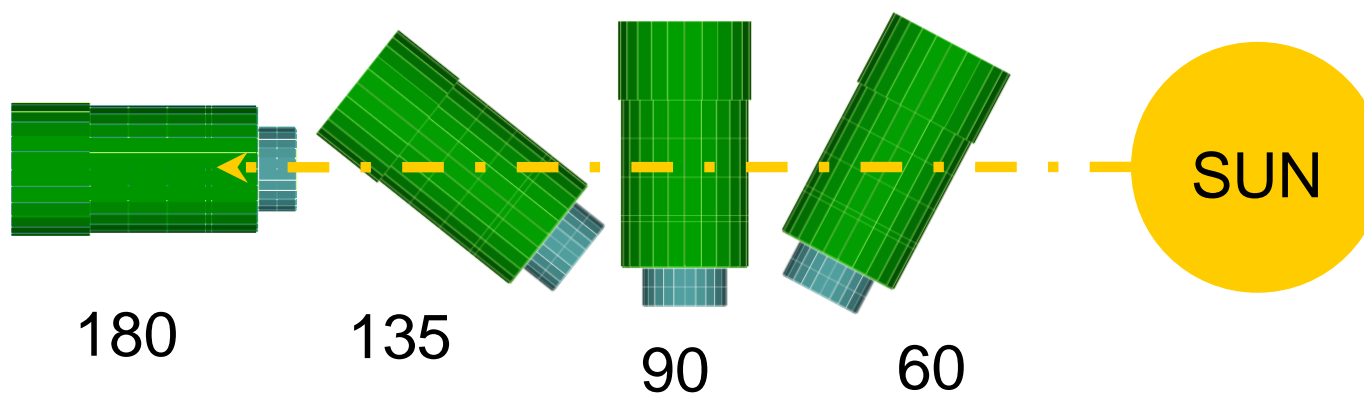

50 layers of MLI \& $60 \mathrm{deg}$ scarfed sun-shade

Cases

Temp
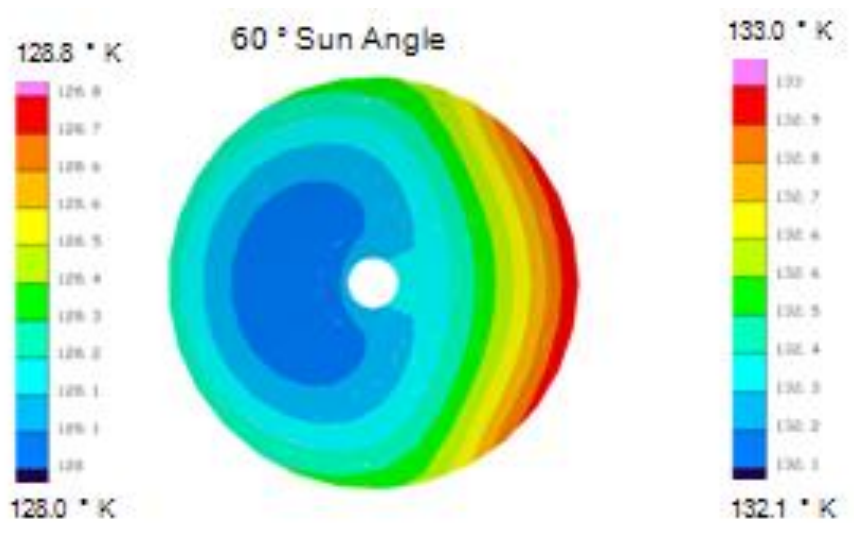

$\begin{array}{rr}60 & 128 \mathrm{~K} \\ 90 & 132 \mathrm{~K} \\ 135 & 141 \mathrm{~K} \\ 180 & 126 \mathrm{~K}\end{array}$
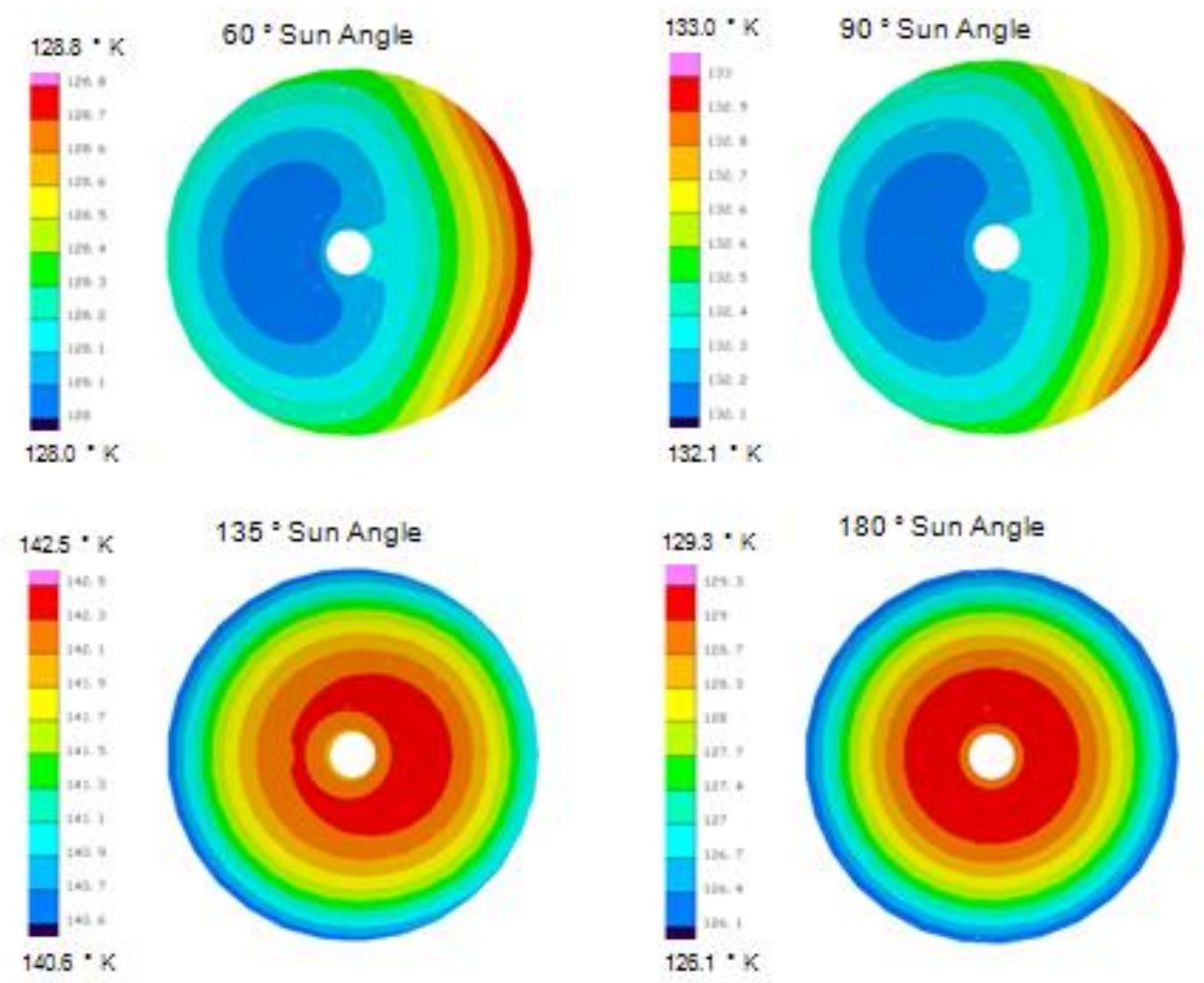


\section{Deg Roll Introduces 0.2K Temp Change}

Gradient changes from 0.9 to $0.7 \mathrm{~K}$ with $500 \mathrm{hr}$ Time Constant.



\section{Deg Slew Introduces 0.2K Temp Change}

Gradient changes from 0.9 to $0.7 \mathrm{~K}$ with $500 \mathrm{hr}$ Time Constant.
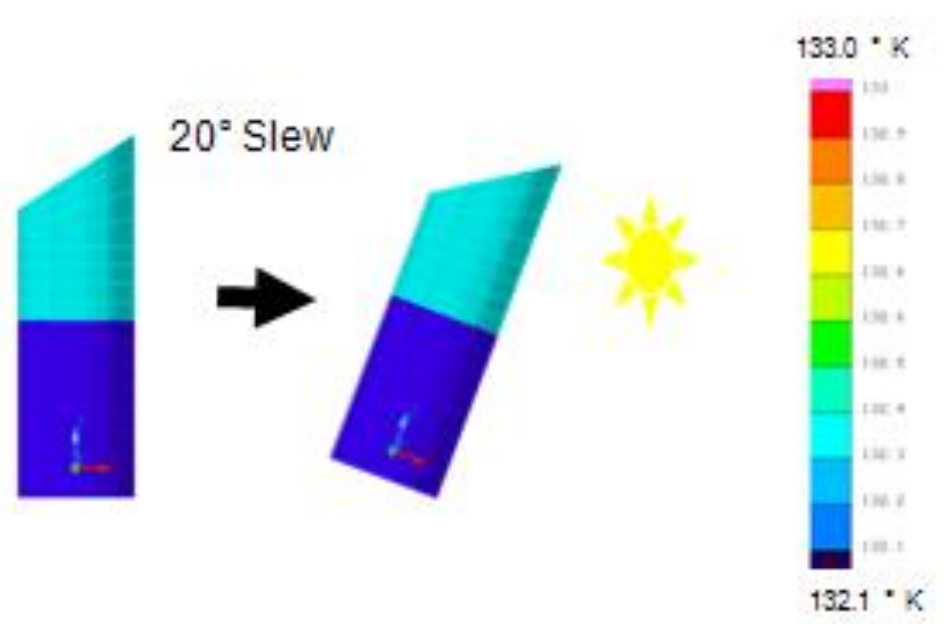

Transient $20^{\circ}$ Slew, Time $=0$ hrs
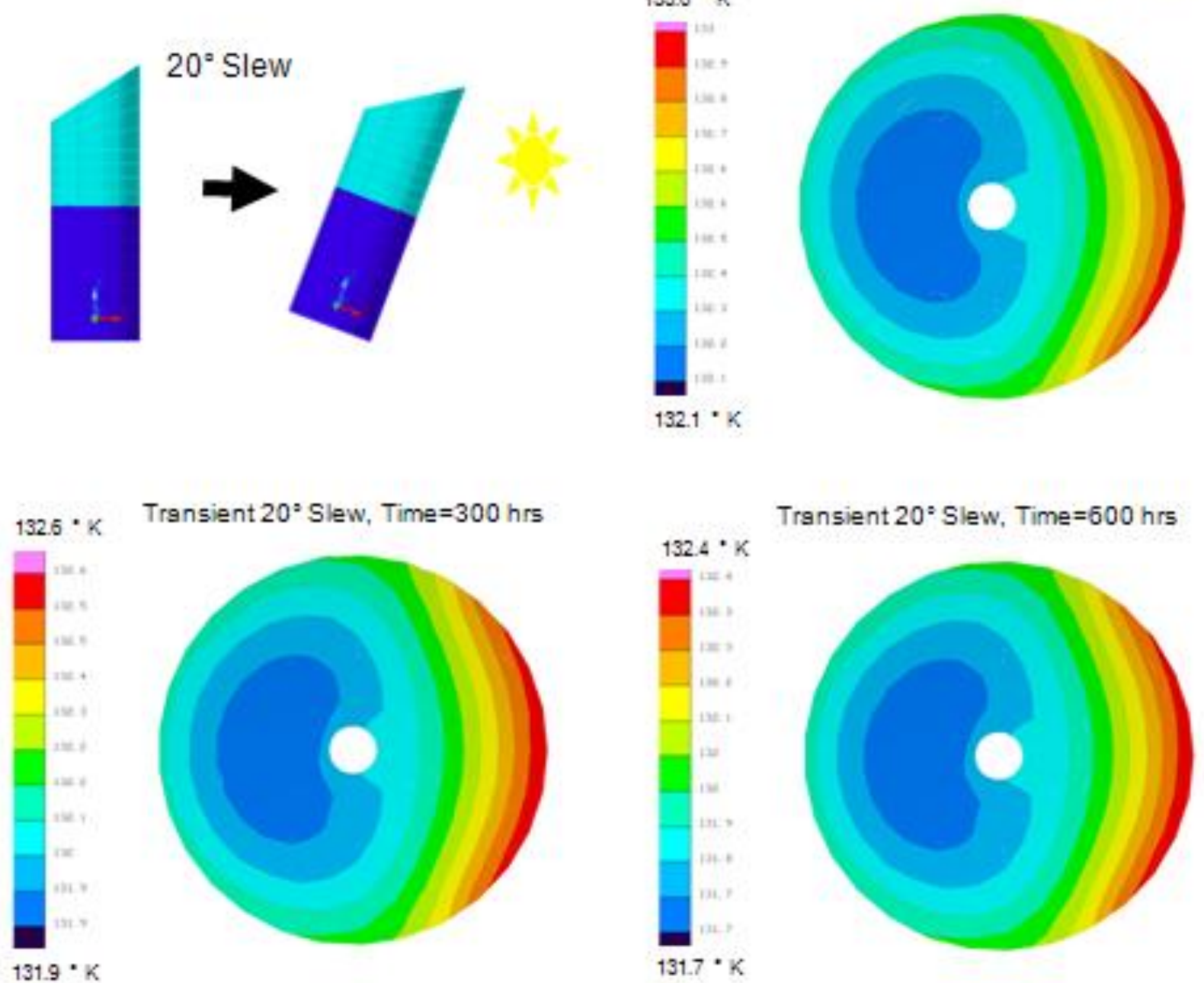

Transient $20^{\circ}$ Slew, Time $=300 \mathrm{hrs}$

Transient $20^{*}$ Slew, Time $=600 \mathrm{hrs}$
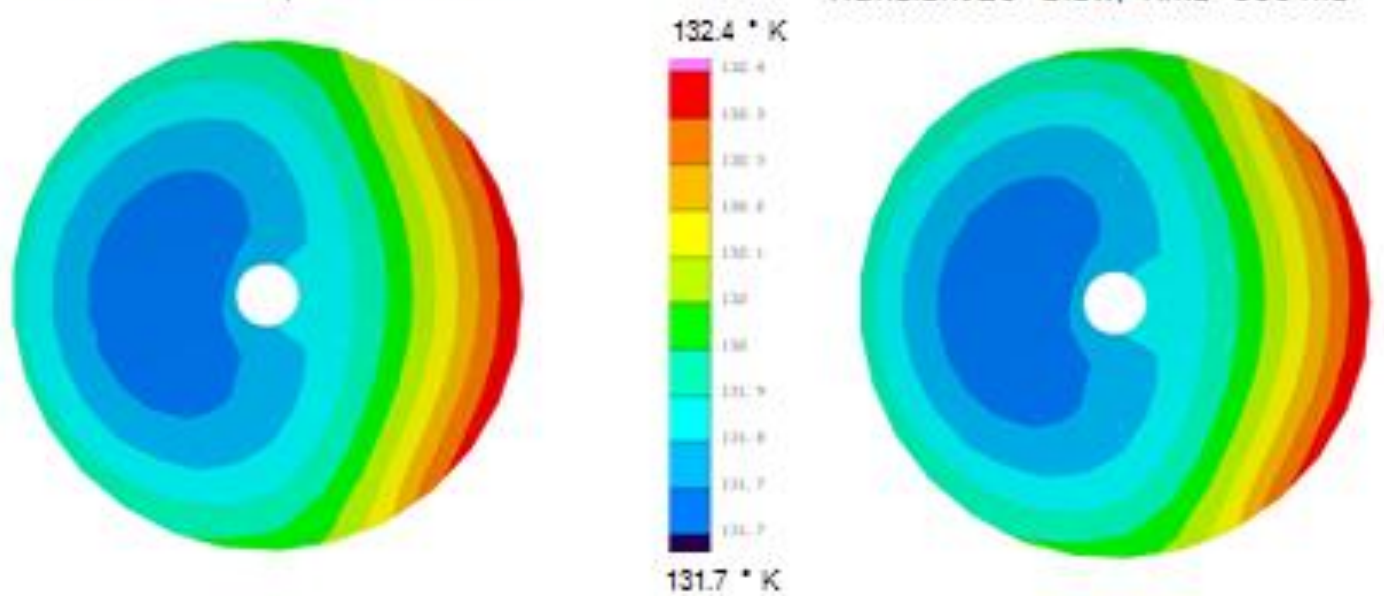


\section{Spacecraft}

Provides all pointing, power, comm, data handling, station keeping, momentum unloading, propulsion \& thermal control.

Packaged in a donut-shaped volume surrounding instrument bay.
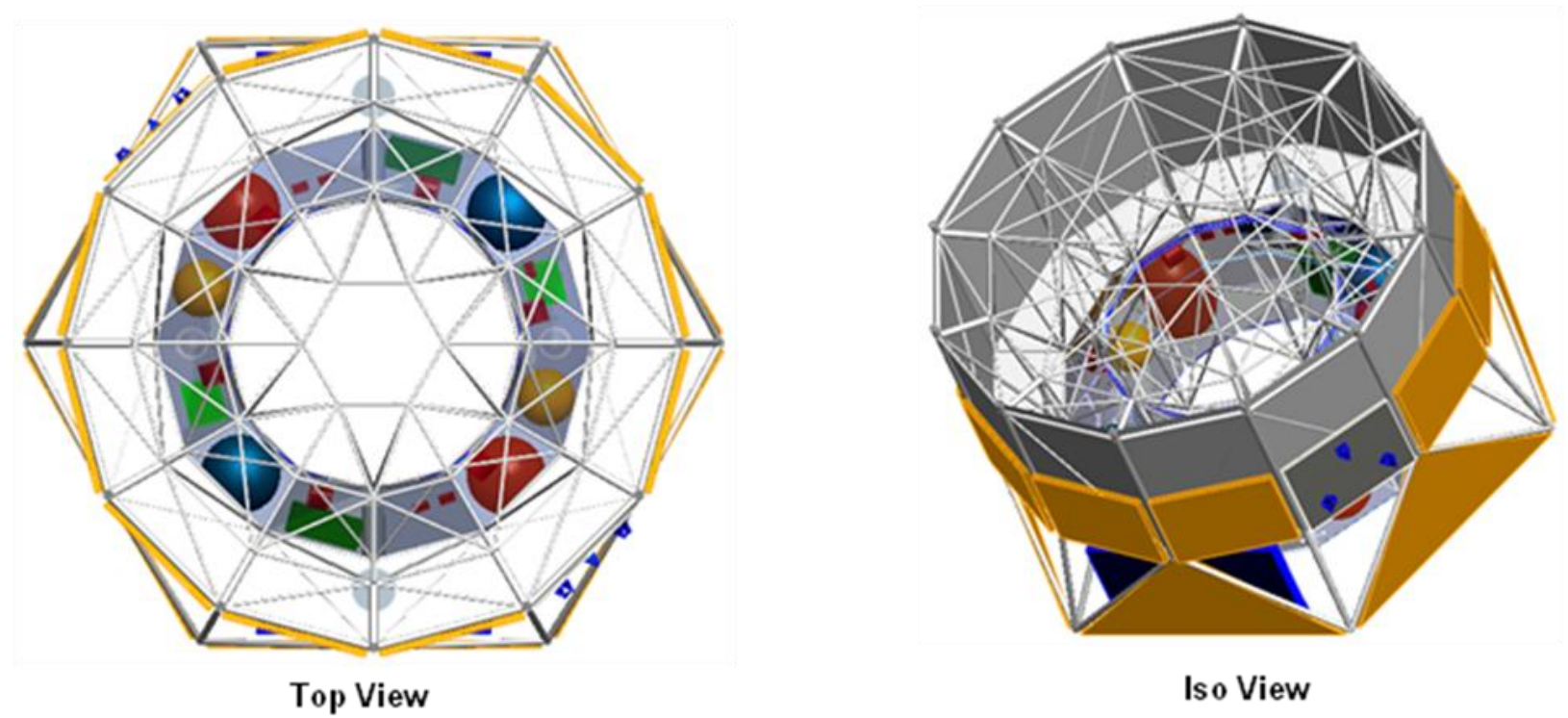

Iso View

Designed for serviceability, key subsystem components are grouped into Orbital Replaceable Units (ORUs) 


\section{Attitude Control System}

ATLAST-8m employs body pointing, similar to HST.

The reason is driven by UV science.

Key requirements

slew 60 degrees in 90 minutes (required) or 40 minutes (desired);

roll about line of sight by 30 degrees in 30 to 60 minutes;

pointing stability of 1.6 mas;

minimum of 4500 minutes continuous observing time 


\section{Attitude Control System}

Star Trackers and Fine Guidance Sensors control reaction wheels and control moment gyros to point the observatory.

Pointing is controlled using guide stars in two FGS modules separated on the sky by 0.5 degree. This separation provides roll control about boresight at a lower bandwidth and with much better stability than the 0.2 mas rms requirement.

Reaction wheels provide momentum storage capability for a minimum of 4500 minutes continuous observation time.

Active Vibration Isolation system between spacecraft and observatory eliminate jitter to achieve sub 1.6 mas stability. 


\section{Science Observation}

Two solar panels on deployable booms balance solar pressure exerted on sunshade to achieve 6.25 days of continuous highprecision pointing observation. By making slight adjustments in boom length, indefinite observation times can be achieved.
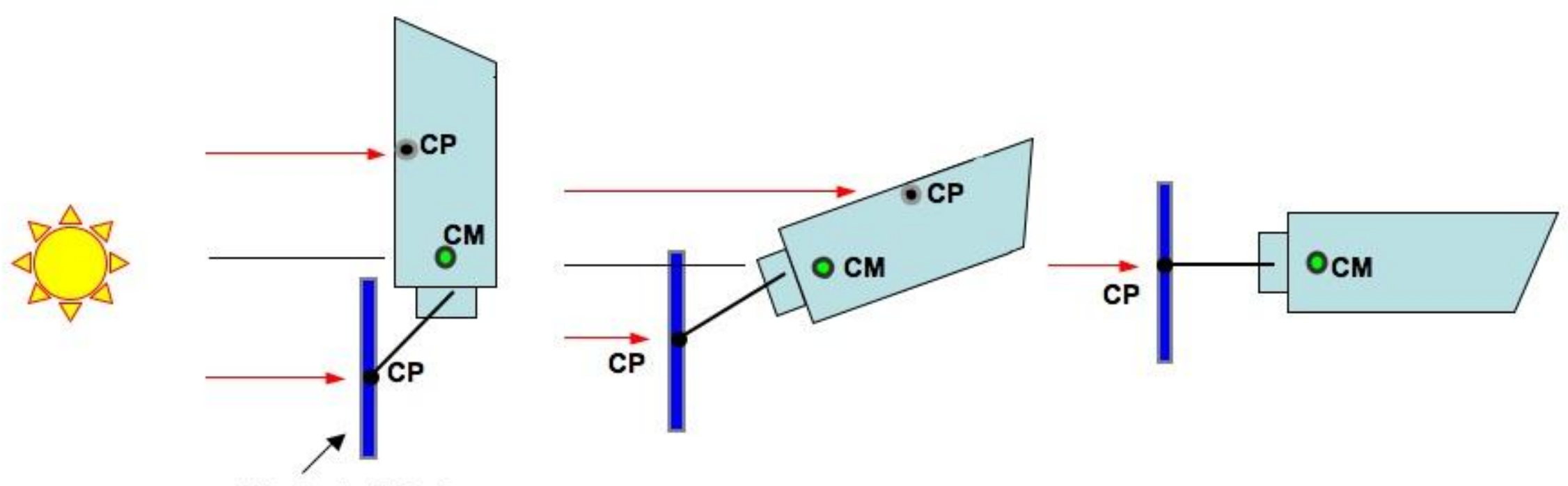

Gimbaled Solar Arrays 


\section{Mass Budget - CBE without Margin}

Since Zerodur is denser than ULE, the most massive possible PM is

$175 \mathrm{~mm}$ thick Zerodur at $25,000 \mathrm{~kg}$.

Total Payload with a 175 $\mathrm{mm}$ Zerodur mirror is estimated at $\sim 50,000 \mathrm{~kg}$ with 30\% margin against Ares V's $65 \mathrm{mt}$ to SE-L2 capacity.

\begin{tabular}{|l|c|c|}
\hline \multicolumn{3}{|c|}{ ATLAST-8m Mass and Power Budget } \\
\hline \multicolumn{1}{|c|}{ Parameter } & Value & Units \\
\hline Mass & & \\
\hline Optical Telescope Assembly & 33,908 & $\mathrm{~kg}$ \\
\hline Primary Mirror Assembly & 24,988 & $\mathrm{~kg}$ \\
\hline Primary Mirror & 19,250 & $\mathrm{~kg}$ \\
\hline \multicolumn{1}{|c|}{ Primary Mirror Mount } & 5,738 & $\mathrm{~kg}$ \\
\hline Secondary Mirror and Aft-Optics Assemblies & 2,118 & $\mathrm{~kg}$ \\
\hline Structure \& Other & 6,802 & $\mathrm{~kg}$ \\
\hline Instruments & 1,789 & $\mathrm{~kg}$ \\
\hline Spacecraft & 4,577 & $\mathrm{~kg}$ \\
\hline Propellant & 4,921 & $\mathrm{~kg}$ \\
\hline TOTAL PAYLOAD & $\mathbf{4 5 , 1 9 5}$ & $\mathbf{k g}$ \\
\hline Launch Vehicle Payload Capacity & 65,000 & $\mathrm{~kg}$ \\
\hline Launch Vehicle Mass Margin (\%) & 44 & $\%$ \\
\hline Payload Mass if Primary Mirror = 25,000 kg & 50,945 & $\mathrm{~kg}$ \\
\hline Launch Vehicle Mass Margin & 14,055 & $\mathrm{~kg}$ \\
\hline Payload Mass without Primary Mirror & 25.045 & $\mathrm{~kg}$ \\
\hline Payload Mass Margin (\%) & 56 & $\%$ \\
\hline Spacecraft Bus Power Requirement & 8,589 & $\mathrm{~W}$ \\
\hline Projected End of Life Solar Panel Production & 11,200 & $\mathrm{~W}$ \\
\hline Solar Panel Power Margin (\%) & 30 & $\%$ \\
\hline
\end{tabular}




\section{Mission Life}

Initial Mission designed for a $5 \mathrm{yr}$ mission life (10 yr goal)

But, there is no reason why mission should end after 5 or 10 years

Hubble has demonstrated the value of on-orbit servicing

The telescope itself could last 30 or even 50 years

L2 Virtual Mountain Top - service \& upgrade observatory robotically using AR\&D (as demonstrated by Orbital Express)

Or, 'propel' from SE-L2 to EM-L1 for Human Servicing. 


\section{Notional Servicing Scenario}

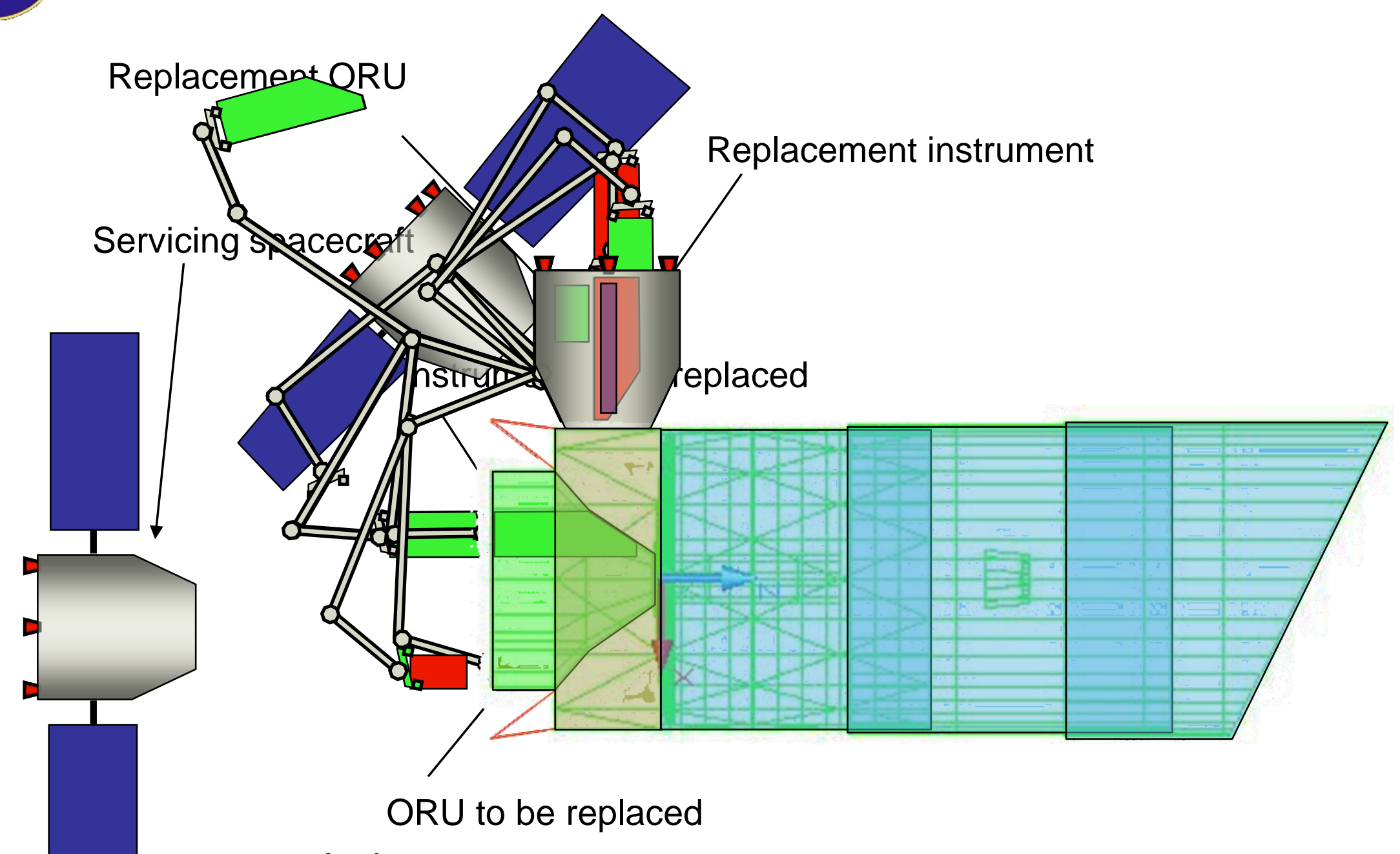

And so on ... 


\section{Conclusion}

ATLAST Astrophysics Mission Concept Study developed a detailed point design for an 8-m monolithic observatory:

- optical design;

- structural (primary mirror support structure, sun shade and secondary mirror support structure);

- thermal analysis;

- spacecraft (structure, propulsion, GN\&C, avionics, power systems and reaction wheels);

- mass and power budgets; and

- cost \& schedule

Complete report and responses to Decadal RFIs can be found at: http://www.stsci.edu/institute/atlast/index html_ATLASTMissio nConceptStudy Page 
$+^{++}$ NASA $\mathrm{ft+}_{++}^{+} \mathrm{J}^{+}$

Any Question?

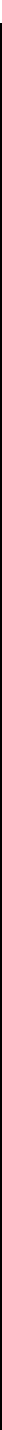

